\title{
The Multi-Sectoral Determinants (linkages) for The Agricultural Total Factor Productivity in Egypt
}

Naglaa Ahmed Mohamed Abdelrahman

Assistant-Lecturer,

Department of Agricultural Economics, Faculty of Agriculture, Cairo University

Dr. Ali Ahmed Ibrahim

Professor, Department of Agricultural Economics

Faculty of Agriculture ,Zagazig University
Dr. Gamal Siam

Professor Emeritus,

Dr. Walid Yehia Sallam

Associate professor, Department of Agricultural

Economics, Faculty of Agriculture, Cairo University

\begin{abstract}
The study aimed at figuring out the sources of growth in the agricultural TFP (Total Factor Productivity) in Egypt. Depending on the multi-sectoral development indicators published in the World Bank about Egypt. This was achieved through: first, illustrating the correlation between the TFP and the groups of development indicators; using the correlation coefficient matrix. Second, selecting the highly correlated indicators with the TFP and conducting multiple regressions. The indicators were divided into 3 groups according to data availability; 54 years (19612014), 44 years (1971-2014), and 25 years (1990-2014). As for the 54-year-analysis, the relationship among the TFP in the Egyptian agricultural sector and each of Gross Domestic Saving (GDS), and official exchange rate (OER) is positive and statistically significant. But, the relationship between the TFP and the Agri-row-material exports (ARMEX) is negative and statistically insignificant. As for the 44-year-analysis, the relationship among the TFP and each of net official development assistance (NODA), electric power consumption (EPC), and imports of goods and services (IMGS) were positive and statistically significant. But, the relationship between the TFP and the fossil fuel energy consumption (FFEC) is negative and statistically insignificant. As for the 25-year-analysis, the relationship among the TFP and each of Research and Development (RD), Rail lines (RL), and foreign direct investment inflows (FDII) were positive and statistically significant. But, the relationship between the TFP and the taxes on exports (TOEX) is negative and statistically significant.
\end{abstract}

\section{Summary}

The study aimed at figuring out the sources of growth in the agricultural TFP in Egypt. Depending on the multi-sectoral development indicators published in the World Bank about Egypt. These sectors were agriculture and rural development, aid effectiveness, climate change, economy and growth, gender, public sector, private sector, poverty, infrastructure, science and technology, public health, social protection and labor, trade, urban development, environment, financial sector, external dept., and finally education. They were used to figure out more explanatory variables the influence the agricultural TFP in Egypt.

\section{This was achieved through:}

- Illustrating the correlation relationship among the TFP and the above mentioned groups of indicators; using the simple correlation coefficient matrix. 
- Selecting the highly correlated indicators with the TFP and conducting an in depth analysis (multiple regression) to get a closer insight into the relation of the agricultural TFP and these multi-sectoral indicators. However, in this step, the indicators were divided into 3 groups according to data availability; 54 years (1961-2014), 44 years (1971-2014), and 25 years (1990-2014).

As for the 54-year-analysis, the relationship among the TFP in the Egyptian agricultural sector and each of Gross Domestic Saving (GDS), and official exchange rate (OER) is positive and statistically significant. But, the relationship between the TFP and the Agri-row-material exports (ARMEX) was negative and statistically insignificant. As for the 44-year-analysis, the relationship among the TFP and the each of net official development assistance (NODA), electric power consumption (EPC), and imports of goods and services (IMGS) were positive and statistically significant. But, the relationship between the TFP and the fossil fuel energy consumption (FFEC) was negative and statistically insignificant. As for the 25-yearanalysis, the relationship among the TFP and the each of Research and Development (RD), Rail lines (RL), and foreign direct investment inflows (FDII) were positive and statistically significant. But, the relationship between the TFP and the taxes on exports (TOEX) was negative and statistically significant.

\section{The study highlights the :}

- importance of Official exchange rate and its relative stability on the TFP growth as well as merchandise imports when it's relatively advanced technology as trade in this case works as a carrier of knowledge.

- Supporting export-oriented-activities that increase the value added, but not the raw materials.

- Redirecting and encouraging the ODA to agriculture, Loans should be kept to its minimum values to limit indebtedness levels and not burden the country with heavy external debts. Better management and coordination of external assistance will increase the positive impact and efficiency of aid system. Raising technical and financial support will improve the performance of projects financed by donors. Also, maximizing the utilization of external financial resources will make positive impacts on the national development capacity of Egypt.

- Fragmented aid that comes in many small slices from a large number of donors creates high transaction costs and makes it difficult for partner countries effectively to manage their own development. Aid fragmentation also increases the risk of duplication and inefficient aid allocation among donors.

- Conducting development plans that are specific to each governorate especially those which receive lower proportions of assistance.

- Infrastructure is not only very important for productivity growth but even triggers it. However, it is important to closely monitor its management and financing. production of electricity.

- Strengthening the role of agricultural institutions and giving high attention to agricultural labor training programs either the domestically funded or the internationally funded. 
- Adopting more efficient use of fossil fuel ; following the maintenance schedules of agricultural machinery, using energy-saving machinery, as well as fixing and constructing viable roads for efficient transport; this idea could be transmitted to rural areas through media alongside with agricultural extension.

- Rural transport services enhancement through: addressing inefficiencies and monopolistic practices of rural transport operators; improving efficiency of overall post-harvest storage and marketing operations; Improve load consolidation practices to reduce costs and increase bargaining power for farmers; Developing modern agricultural supply chains, particularly for high-value export crops and to meet demand of proliferating supermarkets; Increasing resilience to climate impacts, including through rural road improvements; and Establishing farmer's associations or cooperatives to lower the price of transport by arranging and purchasing farm inputs (such as fertilizer) in bulk

- Improving water resources in rural areas through.

1. Ensure water supply for a secure and economically viable agriculture

2. Develop new approaches in agricultural water management

3. Develop pro-poor and affordable agricultural water management

4. Mitigation of environmental and health impacts of new and existing systems

- Adopting foreign and domestic policies that encourages FDI, as it is a strong growth stimulant and redirecting it to give more priority to the agri-labor training as well as the neglected governorates.

- To avoid the negative repercussions of quantitative export restrictions such as export taxes, it would be useful to have in place an improved, multilaterally agreed regulatory framework governing the use of these measures. The alternatives to a conventional export tax: (1) a consumption subsidy, (2) a production tax, and (3) a modification of a conventional export tax that allows additional exports after producers meet a domestic sales requirement.

\section{Introduction}

The agricultural total factor productivity (TFP) growth provides society with opportunities to increase the welfare of people. It is, therefore, worth asking what determinants should policy makers focus on to enhance the performance of the agricultural TFP? This paper attempts to induce the determinants of agricultural productivity growth. It will also investigate to what extent the determinants have implications for policy. Having the calculated TFP as given from the previous work of Abdurrahman, N.et. al. (2018 ). The development indicators (or determinants) considered here are grouped under 18 headings (segments). They were used to figure out more explanatory variables the influence the agricultural TFP in Egypt. These are:

agriculture and rural development, aid effectiveness, climate change, economy and growth, gender, public sector, private sector, poverty, infrastructure, science and technology, public health, social protection and labor, trade, urban development, environment, financial sector, external dept., and finally education. 
The research problem is that there has not been enough work on both areas of agricultural TFP in or the determinants of TFP growth in Egypt. All the previous work has highlighted the need for more studies in both areas.

The research broad objective is to figure out the sources of growth in the agricultural TFP in Egypt. This could be achieved through:

- Illustrating the direction and strength of the correlation relationship among the TFP and the above mentioned groups of indicators.

- Selecting the highly correlated indicators with the TFP and conducting an in depth analysis to get a closer insight into the relation of the agricultural TFP and these multi-sectoral indicators.

\section{Data sources and Methodology:}

\section{Data sources:}

The study depended mainly on the data for the development indicators published in the World Bank. These indicators were categorized into 18 groups as follows: agriculture and rural development, aid effectiveness, climate change, economy and growth, gender, public sector, private sector, poverty, infrastructure, science and technology, public health, social protection and labor, trade, urban development, environment, financial sector, external dept., and finally education.

The agricultural TFP in Egypt was already calculated in the work of Abdurrahman, N. et. al.(2018).

\section{Methodology:}

- The correlation coefficient matrix was applied to illustrate the correlation between the TFP and the above mentioned groups of indicators.

- Each indicator that had a correlation coefficient with the TFP that is greater than or equal to -0.5 or +0.5 was selected for running a multiple regression between agricultural TFP as a dependent variable and all the highly correlated indicators as explanatory variables. However, this second step was executed differently due to data availability of the mentioned indicators. There were divided into 3 groups according to data availability; 54 years (1961-2014), 44 years (1971-2014), and 25 years (1990-2014).

- The stationarity of each indicator in each group of data was tested using Augmented Dickey Fuller (ADF) test Eviews software.

- The serial correlation was tested using Durbin Watson and Breusch-Godfrey Serial Correlation LM Test using Eviews software.

- Heteroskedasticity was tested using Breusch-Pagan-Godfrey test by Eviews software.

\section{1- The correlation coefficient matrix}

A correlation is a number between -1 and +1 that measures the degree of association between two variables. A positive value for the correlation implies a positive association (large values of $\mathrm{X}$ tend to be associated with large values of $\mathrm{Y}$ and small values of $X$ tend to be associated with small values of $Y$ ). A negative value 
for the correlation implies a negative or inverse association (large values of $\mathrm{X}$ tend to be associated with small values of $\mathrm{Y}$ and vice versa). The correlation is computed as:

$$
r=\frac{\sum_{i=1}^{n}\left(X_{i}-\bar{X}\right)\left(Y_{i}-\bar{Y}\right)}{(n-1) S_{X} S_{Y}}
$$

The correlation coefficient measures the strength of a linear relationship between two variables. The correlation coefficient is always between -1 and +1 . The closer the correlation is to $+/-1$, the closer to a perfect linear relationship. Here is how I tend to interpret correlations.

- -1.0 to -0.7 strong negative association.

- -0.7 to -0.3 weak negative association.

- -0.3 to +0.3 little or no association.

- +0.3 to +0.7 weak positive association.

- +0.7 to +1.0 strong positive association.

\section{2- Unit root test, Augmented Dickey Fuller (ADF)}

augmented Dickey-Fuller test (ADF) tests the null hypothesis that a unit root is present in a time seriessample. The alternative hypothesis is different depending on which version of the test is used, but is usually stationarity or trend-stationarity. It is an augmented version of the Dickey-Fuller test for a larger and more complicated set of time series models. The augmented Dickey-Fuller (ADF) statistic, used in the test, is a negative number. The more negative it is, the stronger the rejection of the hypothesis that there is a unit root at some level of confidence. The testing procedure for the ADF test is the same as for the Dickey-Fuller test but it is applied to the model

The unit root test for a variable (Y) can be expressed as follows:

$$
\Delta L Y=B_{1}+B_{2}+B_{3} L Y_{t-1}+u_{t}
$$

We regress the first differences of the $\log$ of $(\mathrm{y})$ on the trend variable and the one-period lagged value of the (y). The null hypothesis is that $\mathrm{B}_{3}$, the coefficient of $\mathrm{LY}_{\mathrm{t}-1}$, is zero.This is called the unit root hypothesis. The alternative hypothesis is: $\mathrm{B}_{3}<0$. A non-rejection of the null hypothesis would suggest that the time series under consideration is non-stationary.

We use the $\tau$ (tau) test, also known as the Dickey-Fuller (DF) test, whose critical values are calculated by simulations and modern statistical packages, such as EVIEWS and STATA.

Add the lagged values of the dependent variable as follows:

$$
\Delta L Y_{t}=B_{1}+B_{2} t+B_{3} L Y_{t-1}+\sum_{i=1}^{m} \alpha_{i} \Delta L Y_{t-i}+\varepsilon_{t}
$$


The DF test can be performed in three different forms:

Random walk : $\Delta L Y_{t}=B_{3} L Y_{t-1}+u_{t}$

Random walk with drift : $\Delta L Y_{t}=B_{1}+B_{3} L Y_{t-1}+u_{t}$

Random walk with drift around a determinis tic trend: $\Delta L Y_{t}=B_{1}+B_{2} t+B_{3} L Y_{t-1}+u_{t}$

- The error term in the Dickey-Fuller test usually has autocorrelation, which needs to be removed if the result is to be valid. The main way is to add lagged dependent variables until the autocorrelation has been mopped up.

- The test is the same as before in that it is the coefficient on the lagged dependent variable that is tested.

- The test is as follows, where the number of lagged dependent variables is determined by an information criteria:

$$
\Delta y_{t}=\beta y_{t-1}+\sum_{i=0}^{N} \Delta y_{t-i}+u_{t}
$$

- When a variable contains two unit roots, it is said to be $I(2)$ and needs to be differenced twice to induce stationarity.

- When using the ADF test, the data is first tested to determine if it contains a unit root, i.e. it is $\mathrm{I}(1)$ and not $\mathrm{I}(0)$

- If it is not I(0), it could be I(1), I(2) or have a higher order of unit roots

- In this case the ADF test needs to be conducted on the differenced variable to determine if it is I(1) or I(2). (It is very rare to find I(3) or higher orders).

- Software: Eviews

1- draw a line graph of the variable to determine with type of randome walk it is (random walk, RW with drift, or RW with trend)

2- view - unit root tests - Augmented Dicky Fuller Test- choose the unit root type

3 - test results interpretation: if the p-value is $>.05$ then it is non- significant then we accept the null hypothesis that there is a unit root.

\section{4- Durbin Watson}

A test that the residuals from a linear regression or multiple regression are independent. Because most regression problems involving time series data exhibit positive autocorrelation, the hypotheses usually considered in the Durbin-Watson test areH $0: p=0, H 1: p=1$, and the test statistic is:

$$
\mathrm{d}=\frac{\sum_{\mathrm{i}=2\left(\mathrm{e}_{\mathrm{i}}-\mathrm{e}_{\mathrm{i}=1}\right)^{2}}^{\sum_{\mathrm{i}=1}^{\mathrm{n}} \mathrm{e}_{\mathrm{i}}^{2}}}{\mathrm{u}^{2}}
$$

whereei $=y i-{ }^{\wedge} y i$ and yi and ${ }^{\wedge}$ yi are, respectively, the observed and predicted values of the response variable for individual $i$. $d$ becomes smaller as the serial correlations increase. Upper and lower critical values, $\mathrm{dU}$ and $\mathrm{dL}$ have been tabulated for different values of $\mathrm{k}$ (the number of explanatory variables) and $\mathrm{n}$. If $\mathrm{d}<\mathrm{dL}$ reject $\mathrm{H} 0$; If $\mathrm{d}>\mathrm{dU}$ do not reject $\mathrm{H} 0$; If $\mathrm{dL}<\mathrm{d}<\mathrm{dU}$ test is inconclusive.

\section{4- Breusch-Godfrey Serial Correlation LM}

The Breusch-Godfrey serial correlation LM test is a test for autocorrelation in the errors in a regression model. It makes use of the residuals from the model being 
considered in a regression analysis, and a test statistic is derived from these. The null hypothesis is that there is no serial correlation of any order up to $p$. The test is more general than the Durbin-Watson statistic (or Durbin's $h$ statistic), which is only valid for nonstochasticregressors and for testing the possibility of a first-order autoregressive model (e.g. AR(1)) for the regression errors. The BG test has none of these restrictions, and is statistically more powerful than Durbin's $h$ statistic.

Consider a linear regression of any form, for example

$$
Y_{t}=\beta_{1}+\beta_{2} X_{t, 1}+\beta_{3} X_{t, 2}+u_{t}
$$

where the errors might follow an $\operatorname{AR}(p)$ autoregressive scheme, as follows:

$$
u_{t}=\rho_{1} u_{t-1}+\rho_{2} u_{t-2}+\cdots+\rho_{p} u_{t-p}+\varepsilon_{t} .
$$

The simple regression model is first fitted by ordinary least squares to obtain a set of sample residuals $\mathrm{u}^{\wedge} \mathrm{t}\left\{\backslash\right.$ displaystyle $\left.\{\text { hat }\{\mathrm{u}\}\}_{-}\{\mathrm{t}\}\right\}$ Breusch and Godfrey proved that, if the following auxiliary regression model is fitted

$$
\hat{u}_{t}=\alpha_{0}+\alpha_{1} X_{t, 1}+\alpha_{2} X_{t, 2}+\rho_{1} \hat{u}_{t-1}+\rho_{2} \hat{u}_{t-2}+\cdots+\rho_{p} \hat{u}_{t-p}+\varepsilon_{t}
$$

and if the usual R2 statistic is calculated for this model, then the following asymptotic approximation can be used for the distribution of the test statistic

$$
n R^{2} \sim \chi_{p}^{2}
$$

when the null hypothesis: $\mathrm{H} 0:\{\mathrm{pi}=0$ for all $\mathrm{i}\}$

holds (that is, there is no serial correlation of any order up to $p$ ). Here $n$ is the number of data-points available for the second regression, that for $\hat{u}_{t}$

$$
n=T-p,
$$

where $T$ is the number of observations in the basic series. Note that the value of $\mathrm{n}$ depends on the number of lags of the error term $(p)$.

Software: Eviews - excute the regression - obtain the results - view - Residual diagnostics - Serial Correlation LM Test.

\section{5- Breusch-Pagan-Godfrey}

the Breusch-Pagan test, developed in 1979 by Trevor Breusch and Adrian Pagan, ${ }^{[1]}$ is used to test for heteroskedasticity in a linear regression model.It tests whether the variance of the errors from a regression is dependent on the values of the independent variables. In that case, heteroskedasticity is present. Suppose that we estimate the regression model

$$
y=\beta_{0}+\beta_{1} x+u,
$$

obtain from this fitted model a set of values for $\hat{u}$ the residuals. Ordinary least squares constrains these so that their mean is 0 and so, given the assumption that their variance does not depend on the independent variables, an estimate of this variance can be obtained from the average of the squared values of the residuals. If the assumption is not held to be true, a simple model might be that the variance is linearly related to independent variables. Such a model can be examined by regressing the squared residuals on the independent variables, using an auxiliary regression equation of the form $\hat{u}^{2}=\gamma_{0}+\gamma_{1} x+v$. 
This is the basis of the Breusch-Pagan test. It is a chi-squared test: the test statistic is distributed $n \chi^{2}$ with $k$ degrees of freedom. If the test statistic has a p-value below an appropriate threshold (e.g. $p<0.05)$ then the null hypothesis of homoskedasticity is rejected and heteroskedasticity assumed.

If the Breusch-Pagan test shows that there is conditional heteroskedasticity, one could either use weighted least squares (if the source of heteroskedasticity is known) or use heteroscedasticity-consistent standard errors.

\section{Procedure:}

Under the classical assumptions, ordinary least squares is the best linear unbiased estimator (BLUE), i.e., it is unbiased and efficient. It remains unbiased under heteroskedasticity, but efficiency is lost. Before deciding upon an estimation method, one may conduct the Breusch-Pagan test to examine the presence of heteroskedasticity. The Breusch-Pagan test is based on models of the type ${ }^{\sigma_{i}^{2}}=h\left(z_{i}^{\prime} \gamma\right)$

for the variances of the observations where $z_{i}=\left(1, z_{2 i}, \ldots, z_{p i}\right)$ explain the difference in the variances. The null hypothesis is equivalent to the (p-1) parameter restrictions: $\gamma_{2}=\cdots=\gamma_{p}=0$.

The following Lagrange multiplier (LM) yields the test statistic for the Breusch-Pagan test

$$
\mathrm{LM}=\left(\frac{\partial \ell}{\partial \theta}\right)^{\top}\left(-E\left[\frac{\partial^{2} \ell}{\partial \theta \partial \theta^{\prime}}\right]\right)^{-1}\left(\frac{\partial \ell}{\partial \theta}\right) .
$$

This test is analogous to following the simple three-step procedure:

Step 1: Apply OLS in the model $y=X \beta+\varepsilon$. and compute the regression residuals

Step 2: Perform the auxiliary regression $e_{i}^{2}=\gamma_{1}+\gamma_{2} z_{2 i}+\cdots+\gamma_{p} z_{p i}+\eta_{i}$. Always, $z$ could be partly replaced by independent variables $x$.

Step 3: The test statistic is the result of the coefficient of determination of the auxiliary regression in Step 2 and sample size with $L M=n R^{2}$.

The test statistic is asymptotically distributed as $\chi_{p-1}^{2}$ under the null hypothesis of homoskedasticity.

Software: Eviews - excute the regression - obtain the results - view - Residual diagnostics - Heteroscedasticity Tests - Breusch-Pagan test - if ( $p$-value) $>0.05$ then we accept the null hypothesis and there is no Heteroscedasticity.

\section{Results and Discussion}

The results consist of two main parts. First part is executing the simple correlation coefficient matrix among the TFP and the development indicators with respect to their sector. Then selecting the indicators that had highly simple correlationcoefficients, i.e., that is above +0.5 or -0.5 . this paves the way for the second part, which is running a multiple regression among the TFP as a dependent variable and the development indicators as explanatory variables. However, the possible multi sectoral determinants of agricultural TFP in Egypt are presented in 
three groups according to data availability and regardless of their sector; 54 years 1961-2014, 44 years 1971-2014, and 25 years 1990-2014.

In this section the simple correlation coefficient matrix was applied to illustrate the correlation between the TFP and the development indicators published in the World Bank. These indicators were categorized into 18 groups as follows: agriculture and rural development, aid effectiveness, climate change, economy and growth, gender, public sector, private sector, poverty, infrastructure, science and technology, public health, social protection and labor, trade, urban development, environment, financial sector, external dept., and finally education.

The agricultural Development segment, there was a relatively strong positive correlation (more than 0.5) among the TFP and Access to electricity, rural 0.95; Agriculture value added per worker 0.98; Agricultural machinery, tractors per 100 sq. km of arable land 0.92; Permanent cropland 0.89; Land under cereal production 0.96; Rural population 0.98. However, there was a relatively strong negative correlation (less than -0.5) among the TFP and Agricultural raw materials imports 0.73; Agricultural raw materials exports -0.53 ; Agriculture, value added ( $\%$ of GDP) -0.91; Employment in agriculture -0.69; Employment in agriculture, male -0.83 . However, there was a relatively weak negative correlation among the TFP and Rural population (\% of total) 0.01 ; Rural population growth -0.38 ; Employment in agriculture, female 0.08 , Table 1 .

\section{Table 1. The simple Correlation coefficient matrix among TFP and agricultural} development indicators.

\begin{tabular}{|c|c|c|c|c|c|c|c|c|c|c|c|c|c|c|c|}
\hline \multicolumn{16}{|l|}{ Agricultural Development } \\
\hline & TFP & 1. & 2. & 3. & 4. & 5. & 6. & 7. & 8. & 9. & 10. & 11. & 12. & 13. & 14. \\
\hline TFP & 1 & & & & & & & & & & & & & & \\
\hline 1. Agricultural raw materials exports (\% of merchandise exports) & -0.53 & 1 & & & & & & & & & & & & & \\
\hline 2. Agricultural raw materials imports $\% \%$ of merchandise imports) & -0.73 & 0.51 & 1 & & & & & & & & & & & & \\
\hline 3. Rural population (\% of total population) & 0.01 & 0.18 & -0.05 & 1 & & & & & & & & & & & \\
\hline 4. Rural population growth (annual \%) & -0.38 & 0.06 & 0.38 & -0.62 & 1 & & & & & & & & & & \\
\hline 5. Rural population & 0.98 & -0.51 & -0.76 & 0.05 & -0.37 & 1 & & & & & & & & & \\
\hline 6. Employment in agriculture (\% of total employment) & -0.69 & 0.23 & 0.52 & -0.46 & 0.53 & -0.72 & 1 & & & & & & & & \\
\hline 7. Employment in agriculture, male (\% of male employment) & -0.83 & 0.36 & 0.63 & -0.16 & 0.46 & -0.85 & 0.86 & 1 & & & & & & & \\
\hline 8. Employment in agriculture, female (\% of female employment) & 0.08 & -0.19 & -0.11 & -0.63 & 0.24 & 0.07 & 0.48 & 0.03 & 1 & & & & & & \\
\hline 9. Agriculture, value added (\% of GDP) & -0.91 & 0.66 & 0.72 & 0.09 & 0.18 & -0.92 & 0.54 & 0.75 & -0.19 & 1 & & & & & \\
\hline 10. Access to electricity, rural (\% of rural population) & 0.95 & -0.44 & -0.76 & 0.13 & -0.54 & 0.96 & -0.75 & -0.85 & 0.02 & -0.82 & 1 & & & & \\
\hline 11. Agriculture value added per worker (constant 2010 us\$) & 0.98 & -0.52 & -0.75 & 0.02 & -0.33 & 1.00 & -0.71 & -0.85 & 0.08 & -0.93 & 0.94 & 1 & & & \\
\hline 12. Agricultural machinery, tractors per 100 sq. km of arable land & 0.92 & -0.59 & -0.70 & 0.09 & -0.46 & 0.92 & -0.64 & -0.73 & 0.02 & -0.84 & 0.94 & 0.91 & 1 & & \\
\hline 13. Permanent cropland (\% of land area) & 0.89 & -0.68 & -0.68 & -0.14 & -0.30 & 0.89 & -0.49 & -0.71 & 0.21 & -0.90 & 0.86 & 0.89 & 0.92 & 1 & \\
\hline 14. Land under cereal production (hectares) & 0.96 & -0.60 & -0.71 & 0.06 & -0.35 & 0.95 & -0.64 & -0.81 & 0.13 & -0.94 & 0.91 & 0.95 & 0.91 & 0.91 & 1 \\
\hline
\end{tabular}

Source: Author's calculations using MS.Excel software.

Aid Effectiveness segment, there was a relatively strong positive correlation among the TFP and Net ODA received per capita 0.51 ; Technical cooperation grants 
-0.89 . On the other hand, there was a relatively weak negative correlation among the TFP and Grants, excluding technical cooperation -0.18, Table 2.

Table 2. The simple Correlation coefficient matrix among TFP and aid effectiveness indicators.

\begin{tabular}{|llllll|}
\hline \multicolumn{2}{|l|}{ aid Effectiveness } & TFP & 1. & 2. & 3. \\
\cline { 2 - 7 } & TFP & $\mathbf{1}$ & & & \\
1. & Net ODA received per capita (current US\$) & 0.51 & 1 & & \\
2. & Technical cooperation grants (BoP, current US\$) & -0.89 & 0.46 & 1 & \\
3. & Grants, excluding technical cooperation (BoP, current US\$) & -0.18 & 0.89 & 0.12 & 1 \\
\hline
\end{tabular}

Source: Author's calculations using MS.Excel software.

Climate Change segment, there was a relatively strong positive correlation among the TFP and Improved water source (\% of population with access) 0.99 ; Energy use 0.93; Electric power consumption 0.98; Electricity production from renewable sources, excluding hydroelectric $\%$ total 0.92 ; Electricity production from renewable sources, excluding hydroelectric $(\mathrm{kWh}) 0.90$; Access to electricity 0.95 . However, there was a relatively strong negative correlation among the TFP and Mortality rate -0.95 ; Electricity production from oil sources -0.68 ; Renewable energy consumption -0.94. On the other hand, there was a relatively weak positive correlation among the TFP and Foreign direct investment, net inflows (\% of GDP) 0.32 . And, there was a relatively weak negative correlation among the TFP and Population growth -0.12 , Table 3 .

Table 3. The simple Correlation coefficient matrix among TFP and climate change indicators.

\begin{tabular}{|c|c|c|c|c|c|c|c|c|c|c|c|c|}
\hline \multicolumn{13}{|l|}{ Climate Change } \\
\hline & TFP & 1 & 2 & 3. & 4. & 5 & 6. & 7. & 8. & g. & 10. & 11. \\
\hline$\overline{\text { TFP }}$ & 1 & & & & & & & & & & & \\
\hline 1. Population growth (annual \%) & -0.12 & 1 & & & & & & & & & & \\
\hline 2 Improved water source (\% of population with access) & 0.99 & -0.15 & 1 & & & & & & & & & \\
\hline 3. Mortality rate, under-5(per 1,000 live births) & -0.95 & 0.35 & -0.97 & 1 & & & & & & & & \\
\hline 4. Energy use (kg of oil equivalent per capita) & 0.93 & -0.14 & 0.92 & -0.87 & 1 & & & & & & & \\
\hline 5. Electric power consumption (kWh per capita) & 0.98 & -0.05 & 0.99 & -0.93 & 0.96 & 1 & & & & & & \\
\hline 6. Renewable energy consumption (\% of total final energy consun & -0.94 & 0.12 & -0.93 & 0.89 & -0.97 & -0.95 & 1 & & & & & \\
\hline 7. Electricity production from renewable sources, excluding hydro & 0.92 & 0.05 & 0.93 & -0.85 & 0.93 & 0.97 & -0.93 & 1 & & & & \\
\hline 8. Electricity production from renewable sources, excluding hydro & 0.90 & 0.17 & 0.91 & -0.80 & 0.89 & 0.95 & -0.88 & 0.98 & 1 & & & \\
\hline 9. Electricity production from oil sources (\% of total) & -0.68 & 0.31 & -0.75 & 0.76 & -0.70 & -0.73 & 0.64 & -0.71 & -0.66 & 1 & & \\
\hline 10. Access to electricity (\% of population) & 0.95 & -0.32 & 0.97 & -0.99 & 0.90 & 0.94 & -0.91 & 0.87 & 0.82 & -0.76 & 1 & \\
\hline 11. Foreign direct investment, net inflows (\% of GDP) & 0.32 & -0.44 & 0.29 & -0.32 & 0.51 & 0.31 & -0.43 & 0.27 & 0.18 & -0.32 & 0.36 & 1 \\
\hline
\end{tabular}

Source: Author's calculations using MS.Excel software.

Economy Growth segment, there was a relatively strong positive correlation among the TFP and Gross domestic savings 0.92; Gross capital formation 0.93; Gross national expenditure 0.89; Imports of goods and services 0.94; Price level ratio 
of PPP conversion factor (GDP) to market exchange rate 0.65 . However, there was a relatively strong negative correlation among the TFP and Agriculture, value added (\% of GDP) -0.91; Trade in services -0.53 . On the other hand, there was a relatively weak positive correlation among the TFP and Adjusted savings: natural resources depletion 0.02; Adjusted savings: energy depletion 0.00; Agriculture, value added annual $\%$ growth 0.19 . And, there was a relatively weak negative correlation among the TFP and Terms of trade adjustment -0.16 ; Net taxes on products -0.07 ; Net taxes on products -0.16 ; GNI growth -0.05 ; GDP per capita growth -0.01 ; GDP growth 0.02; Trade (\% of GDP) -0.09; Inflation, consumer prices -0.10 ; Food, beverages and tobacco ( $\%$ of value added in manufacturing) -0.18 , Table 4.

Table 4. The simple Correlation coefficient matrix among TFP and economy growth indicators.

\begin{tabular}{|c|c|c|c|c|c|c|c|c|c|c|c|c|c|c|c|c|c|c|c|c|}
\hline \multicolumn{21}{|l|}{ Economy Growth } \\
\hline & TFP & 1. & 2. & 3. & 4. & 5. & 6. & 7. & 8. & 9. & 10. & 11. & 12. & 13. & 14. & 15. & 16. & 17. & 18. & 19. \\
\hline TFP & 1 & & & & & & & & & & & & & & & & & & & \\
\hline 1. Price level ratio of PPP conversion factor (GDP) to market ex & 0.65 & 1 & & & & & & & & & & & & & & & & & & \\
\hline 2. Terms of trade adjustment (constant LCU) & -0.16 & -0.50 & 1 & & & & & & & & & & & & & & & & & \\
\hline 3. Net taxes on products (current LCU) & -0.07 & -0.40 & 0.09 & 1 & & & & & & & & & & & & & & & & \\
\hline 4. Net taxes on products (current US\$) & -0.16 & -0.37 & 0.01 & 0.98 & 1 & & & & & & & & & & & & & & & \\
\hline 5. GNI growth (annual \%) & -0.05 & -0.22 & 0.31 & 0.19 & 0.21 & 1 & & & & & & & & & & & & & & \\
\hline 6. Gross domestic savings (current LCU) & 0.92 & 0.58 & -0.10 & 0.14 & 0.02 & -0.09 & 1 & & & & & & & & & & & & & \\
\hline 7. GDP per capita growth (annual \%) & -0.01 & -0.23 & 0.06 & 0.45 & 0.49 & 0.86 & 0.02 & 1 & & & & & & & & & & & & \\
\hline 8. GDP growth (annual \%) & -0.02 & -0.21 & 0.04 & 0.41 & 0.44 & 0.86 & 0.01 & 1.00 & 1 & & & & & & & & & & & \\
\hline 9. Adjusted savings: natural resources depletion (\% of GN) & 0.02 & -0.30 & 0.40 & 0.04 & -0.10 & 0.19 & 0.18 & 0.14 & 0.19 & 1 & & & & & & & & & & \\
\hline 10. Adjusted savings: energy depletion (\% of GNI) & 0.00 & -0.32 & 0.41 & 0.04 & -0.10 & 0.19 & 0.16 & 0.14 & 0.20 & 1.00 & 1 & & & & & & & & & \\
\hline 11. Food, beverages and tobacco $\%$ of value added in manufact & -0.18 & -0.67 & 0.26 & 0.72 & 0.67 & 0.31 & -0.10 & 0.54 & 0.50 & 0.22 & 0.24 & 1 & & & & & & & & \\
\hline 12. Agriculture, value added (\% of GDP) & -0.91 & -0.64 & 0.04 & 0.33 & 0.43 & 0.06 & -0.84 & 0.13 & 0.13 & -0.06 & -0.04 & 0.36 & 1 & & & & & & & \\
\hline 13. Agriculture, value added (annual \% growth) & 0.19 & 0.12 & -0.16 & 0.28 & 0.35 & 0.28 & 0.11 & 0.39 & 0.35 & -0.47 & -0.46 & 0.26 & -0.01 & 1 & & & & & & \\
\hline 14. Trade (\% of GDP) & -0.09 & -0.62 & 0.49 & 0.36 & 0.23 & 0.39 & 0.08 & 0.36 & 0.37 & 0.70 & 0.70 & 0.54 & 0.02 & -0.27 & 1 & & & & & \\
\hline 15. Gross capital formation (current LCU) & 0.93 & 0.79 & -0.25 & -0.21 & -0.30 & -0.17 & 0.92 & -0.14 & -0.14 & 0.07 & 0.04 & -0.38 & -0.92 & 0.05 & -0.14 & 1 & & & & \\
\hline 16. Gross national expenditure (current LCU) & 0.89 & 0.82 & -0.27 & -0.40 & -0.47 & -0.25 & 0.82 & -0.27 & -0.26 & 0.01 & -0.01 & -0.51 & -0.92 & -0.01 & -0.26 & 0.97 & 1 & & & \\
\hline 17. Inflation, consumer prices (annual \%) & -0.10 & -0.16 & 0.07 & -0.08 & -0.18 & -0.01 & 0.09 & -0.06 & 0.00 & 0.65 & 0.63 & 0.01 & -0.04 & -0.62 & 0.62 & 0.07 & 0.03 & 1 & & \\
\hline 18. Imports of goods and services (BOP, current US\$) & 0.94 & 0.71 & -0.15 & -0.14 & -0.24 & -0.03 & 0.94 & -0.01 & -0.01 & 0.16 & 0.14 & -0.29 & -0.92 & 0.07 & 0.00 & 0.98 & 0.93 & 0.13 & 1 & \\
\hline 19. Trade in senices (\% of GDP) & -0.53 & -0.88 & 0.57 & 0.29 & 0.22 & 0.38 & -0.40 & 0.32 & 0.32 & 0.49 & 0.51 & 0.57 & 0.40 & -0.28 & 0.83 & -0.58 & -0.64 & 0.41 & -0.47 & 1 \\
\hline
\end{tabular}

Source: Author's calculations using MS.Excel software.

Education segment, there was a relatively strong positive correlation among the TFP and Labor force, total $\quad 0.98$. However, there was a relatively strong negative correlation among the TFP and Government expenditure on education, total (\% of GDP) -0.66. On the other hand, there was a relatively weak positive correlation among the TFP and Unemployment, total (\% of total labor force) 0.41 , Table 5.

Table 5. The simple Correlation coefficient matrix among TFP and education indicators.

\begin{tabular}{lrrrr}
\hline Education & & & & 3FP \\
& 1. & 2. & 3. \\
\hline TFP & 0.41 & 1 & & \\
1. Unemployment, total (\% of total labor force) & 0.98 & 0.50 & 1 & \\
2. Labor force, total & -0.66 & -0.29 & -0.70 & 1 \\
3. Government expenditure on education, total (\% of GDP) &
\end{tabular}

Source: Author's calculations using MS.Excel software. 
Energy segment, there was a relatively strong negative correlation (more than 0.5 ) among the TFP and Fossil fuel energy consumption -0.93. On the other hand, there was a relatively weak positive correlation among the TFP and Total natural resources rents 0.20 , Table 6 .

Table 6. The simple Correlation coefficient matrix among TFP and Energy indicators.

\begin{tabular}{lrrr}
\hline Energy & & & \\
& TFP & \multicolumn{1}{c}{ 1. } & 2. \\
\hline TFP & 1 & & \\
1. Total natural resources rents (\% of GDP) & 0.20 & 1 & \\
2. Fossil fuel energy consumption (\% of total) & -0.93 & 0.43 & 1.00 \\
\hline
\end{tabular}

Source: Author's calculations using MS.Excel software.

External Debt Segment, there was a relatively strong positive correlation among the TFP and Official exchange rate 0.90; Foreign direct investment, net inflows 0.60; Foreign direct investment, net outflows 0.56 . However, there was a relatively strong negative correlation among the TFP and Lending interest rate 0.84.And, there was a relatively weak negative correlation among the TFP and Inflation, consumer prices -0.10 , Table 7 .

Table 7. The simple Correlation coefficient matrix among TFP and external debt indicators.

\section{External Debt.}

\begin{tabular}{lrrrrrr} 
& TFP & \multicolumn{1}{c}{ 1. } & 2. & 3. & 4. & 5. \\
\hline TFP & 1 & & & & & \\
Official exchange rate (LCU per US\$, period average) & 0.90 & 1 & & & & \\
2. Lending interest rate (\%) & -0.84 & -0.73 & 1 & & & \\
3. Inflation, consumer prices (annual \%) & -0.10 & -0.09 & 0.47 & 1 & & \\
4. Foreign direct investment, net inflows (BoP, current US\$) & 0.60 & 0.53 & -0.47 & 0.16 & 1 & \\
5. Foreign direct investment, net outflows (BoP, current US\$) & 0.56 & 0.40 & -0.44 & 0.40 & 0.63 & 1 \\
\hline
\end{tabular}

Source: Author's calculations using MS.Excel software.

Gender segment, there was a relatively strong negative correlation among the TFP and Employment in agriculture, male (\% of male employment) -0.87. On the other hand, there was a relatively weak positive correlation among the TFP and Employment in agriculture, female (\% of female employment) 0.05 , Table 8.

Table 8. The simple Correlation coefficient matrix among TFP and gender indicators.

\begin{tabular}{lrrr}
\hline Gender & & & \\
& TFP & 1. & 2. \\
\hline TFP & 1 & & \\
1. Employment in agriculture, male (\% of male employment) & -0.87 & 1 & \\
2. Employment in agriculture, female (\% of female employment) & 0.05 & 0.02 & 1 \\
\hline
\end{tabular}

Source: Author's calculations using MS.Excel software. 
Infrastructure segment, there was a relatively strong positive correlation among the TFP and Improved water source0.99; Improved water source, rural 0.98; Rail lines 0.86; Air transport, freight 0.75; Electric power consumption 0.98, Table 9.

\section{Table 9. The simple Correlation coefficient matrix among TFP and infrastructure indicators.}

\begin{tabular}{|c|c|c|c|c|c|c|}
\hline \multicolumn{7}{|l|}{ Infrastructure } \\
\hline & TFP & 1. & 2. & 3. & 4. & 5. \\
\hline TFP & 1 & & & & & \\
\hline 1. Improved water source (\% of population with access) & 0.99 & 1 & & & & \\
\hline 2. Improved water source, rural (\% of rural population with access & 0.98 & 1.00 & 1 & & & \\
\hline 3. Rail lines (total route-km) & 0.86 & 0.87 & 0.87 & 1 & & \\
\hline 4. Air transport, freight (million ton-km) & 0.75 & 0.81 & 0.81 & 0.73 & 1 & \\
\hline 5. Electric power consumption (kWh per capita) & 0.98 & 0.99 & 0.99 & 0.83 & 0.77 & 1 \\
\hline
\end{tabular}

Source: Author's calculations using MS.Excel software.

Health segment, there was a relatively strong positive correlation among the TFP and Improved sanitation facilities, rural 0.97 ; Health expenditure, private $(\%$ of GDP) 0.74; Health expenditure per capita, PPP 0.98; Health expenditure per capita (current US\$) 0.90; Health expenditure, total (\% of GDP) 0.71 ; Health expenditure, public (\% of GDP) 0.56 . However, there was a relatively strong negative correlation among the TFP and Health expenditure, public (\% of total health expenditure) -0.74 ; Depth of the food deficit -0.57 . On the other hand, there was a relatively weak positive correlation among the TFP and Health expenditure, public ( $\%$ of government expenditure) 0.03, Table 10 .

Table 10. The simple Correlation coefficient matrix among TFP and health indicators.

\begin{tabular}{lrrrrrrrrrr}
\hline Health & \multicolumn{1}{l}{ TFP } & 1. & 2. & 3. & 4. & 5. & 6. & 7. & 8. & 9. \\
\hline TFP & 1 & & & & & & & & \\
1. Improved sanitation facilities, rural (\% of rural population with & 0.97 & 1 & & & & & & & \\
2. Health expenditure, total (\% of GDP) & 0.71 & 0.75 & 1 & & & & & & \\
3. Health expenditure, public \% of GDP) & 0.56 & 0.62 & 0.93 & 1 & & & & & \\
4. Health expenditure, public (\% of government expenditure) & 0.03 & 0.10 & 0.60 & 0.63 & 1 & & & & \\
5. Health expenditure, public \% of total health expenditure) & -0.74 & -0.75 & -0.83 & -0.57 & -0.40 & 1 & & & \\
6. Health expenditure, private (\% of GDP) & 0.74 & 0.77 & 0.99 & 0.87 & 0.56 & -0.90 & 1 & & \\
7. Health expenditure per capita, PPP (constant 2011 international & 0.98 & 0.96 & 0.77 & 0.64 & 0.09 & -0.75 & 0.79 & 1 & \\
8. Health expenditure per capita (current US\$) & 0.90 & 0.84 & 0.61 & 0.44 & 0.02 & -0.67 & 0.65 & 0.93 & 1 \\
9. Depth of the food deficit (kilocalories per person per day) & -0.57 & -0.52 & -0.70 & -0.49 & -0.33 & 0.78 & -0.76 & -0.66 & -0.73 & 1 \\
\hline
\end{tabular}

Source: Author's calculations using MS.Excel software.

Private Sector segment, there was a relatively strong positive correlation among the TFP and Subsidies and other transfers 0.88. However, there was a relatively strong negative correlation among the TFP and Net lending $(+) /$ net borrowing (-) (\% of GDP) -0.84 , Table 11. 
Table 11. The simple Correlation coefficient matrix among TFP and private sector indicators.

\section{Private Sector}

TFP

1. Subsidies and other transfers (current LCU)

2. Net lending (+) / net borrowing (-) (\% of GDP)
TFP

1

0.88

$-0.84$
1.

2.

1

1
-0.70

Source: Author's calculations using MS.Excel software.

Public Sector segment, there was a relatively strong positive correlation among the TFP and GDP per person employed 0.98; Fuel imports 0.89; Research and development expenditure 0.78. However, there was a relatively strong negative correlation among the TFP and Food imports -0.78; Agricultural raw materials imports -0.73; Employment in agriculture (\% of total employment) -0.69 ; Trade in services -0.53 . On the other hand, there was a relatively weak positive correlation among the TFP and Unemployment, total 0.41; Unemployment, male 0.49; Unemployment, female 0.11. And, there was a relatively weak negative correlation among the TFP and Vulnerable employment -0.09, Table 12.

\section{Table 12. The simple Correlation coefficient matrix among TFP and public} sector indicators.

\begin{tabular}{|c|c|c|c|c|c|c|c|c|c|c|c|c|}
\hline Public Sector & & & & & & & & & & & & \\
\hline & TFP & 1. & 2. & 3. & 4. & 5. & 6. & 7. & 8. & g. & 10. & 11. \\
\hline TFP & 1 & & & & & & & & & & & \\
\hline 1. Fuel imports (\% of merchandise imports) & 0.89 & 1 & & & & & & & & & & \\
\hline 2. Food imports (\% of merchandise imports) & -0.78 & -0.76 & 1 & & & & & & & & & \\
\hline 3. Agricultural raw materials imports (\% of merchandise imports) & -0.73 & -0.71 & 0.73 & 1 & & & & & & & & \\
\hline 4. Trade in services (\% of GDP) & -0.53 & -0.36 & 0.18 & 0.36 & 1 & & & & & & & \\
\hline 5. Research and development expenditure (\% of GDP) & 0.78 & 0.70 & -0.51 & -0.58 & -0.61 & 1 & & & & & & \\
\hline 6. Unemployment, total (\% of total labor force) (modeled ILO esti & 0.41 & 0.43 & -0.14 & -0.35 & -0.30 & 0.68 & 1 & & & & & \\
\hline 7. Unemployment, male (\% of male labor force) (modeled ILO est & 0.49 & 0.50 & -0.16 & -0.39 & -0.43 & 0.74 & 0.96 & 1 & & & & \\
\hline 8. Unemployment, female (\% of female labor force) (modeled ILC & 0.11 & 0.14 & -0.04 & -0.15 & 0.00 & 0.27 & 0.77 & 0.58 & 1 & & & \\
\hline 9. GDP per person employed (constant 2011 PPP \$) & 0.98 & 0.87 & -0.71 & -0.77 & -0.64 & 0.82 & 0.46 & 0.55 & 0.15 & 1 & & \\
\hline 10. Vulnerable employment, tota (\% of total employment) & -0.09 & -0.06 & -0.09 & 0.21 & 0.52 & 0.03 & 0.12 & 0.09 & 0.03 & -0.22 & 1 & \\
\hline 11. Employment in agriculture (\% of total employment) & -0.69 & -0.55 & 0.52 & 0.52 & 0.72 & -0.47 & -0.20 & -0.31 & -0.04 & -0.73 & 0.47 & \\
\hline
\end{tabular}

Source: Author's calculations using MS.Excel software.

Trade segment, there was a relatively strong positive correlation among the TFP and Commercial service imports 0.95; Merchandise exports 0.93; Insurance and financial services 0.92; Merchandise imports 0.90; Fuel imports 0.89; Transport services 0.73 ; International tourism, 0.89 ;. However, there was a relatively strong negative correlation among the TFP and Tariff rate, applied, simple mean, all products -0.89; Food imports -0.78; Agricultural raw materials imports -0.73; Share of tariff lines with specific rates, primary products -0.68 ; Taxes on exports -0.63 ; 
Food exports -0.61 Tariff rate, applied, weighted mean, primary products -0.64 ; Trade in services -0.53; Agricultural raw materials exports -0.53 . On the other hand, there was a relatively weak positive correlation among the TFP and Manufactures exports 0.25; Insurance and financial services 0.25 ; Travel services 0.06 . And, there was a relatively weak negative correlation among the TFP and Manufactures imports -0.39 ; Tariff rate, most favored nation, weighted mean, primary products -0.47 ; Trade $(\%$ of GDP) -0.09, Table 13.

\section{Table 13. The simple Correlation coefficient matrix among TFP and trade indicators.}

\begin{tabular}{|c|c|c|c|c|c|c|c|c|c|c|c|c|c|c|c|c|c|c|c|c|c|c|c|c|c|}
\hline \multicolumn{26}{|l|}{$\begin{array}{c}\text { Trade } \\
\end{array}$} \\
\hline & $\pi P p$ & 1 & 2 & 3 & 4 & 5 & 5 & 6 & . & 8. & 2 & & 10 & 11 & 12 & 13. & 14 & 15 & 16 & 17. & 18 & 19. & 20 & 21 & 22 \\
\hline TFP & 1 & & & & & & & & & & & & & & & & & & & & & & & & \\
\hline 1 Merchandise exports (current USS) & 0.93 & 1 & & & & & & & & & & & & & & & & & & & & & & & \\
\hline 2 Manufactures exports (\% of merchandise exports) & 0.25 & 0.33 & 1 & & & & & & & & & & & & & & & & & & & & & & \\
\hline 3 Insuriance and financial services (\% of commercial service expotts) & 0.25 & 0.31 & 0.24 & & 1 & & & & & & & & & & & & & & & & & & & & \\
\hline 4 Food exports (\% of merchandise expots) & .0 .61 & 0.11 & 0.3 & 0.25 & & 1 & & & & & & & & & & & & & & & & & & & \\
\hline s Agriculturalal raw materials exports (\% of merchandise expots) & .0 .53 & 0.62 & 0.09 & 0.09 & & 0.36 & 1 & & & & & & & & & & & & & & & & & & \\
\hline a Travel services (\% of conmercial service imports) & 0.06 & -0.66 & 0.00 & 0.15 & & 0.05 & .0 .08 & 1 & & & & & & & & & & & & & & & & & \\
\hline 7. Transport services (\%of commercial service imports) & 0.73 & 0.82 & 0.22 & 0.02 & & 0.56 & .0 .46 & .0 .36 & 1 & & & & & & & & & & & & & & & & \\
\hline 8 Conmercial service imports (current ISS) & 0.95 & 0.96 & 0.29 & 0.36 & & 0.64 & .0 .63 & 0.02 & 0.13 & & 1 & & & & & & & & & & & & & & \\
\hline q Merchandise imports(current USS) & 0.90 & 0.97 & 0.48 & 0.3 & & 0.78 & .0 .57 & 0.03 & 0.80 & 0.9 & 95 & 1 & & & & & & & & & & & & & \\
\hline 10. Manufiactures imports $\% \%$ of merchandisis imports) & .039 & .025 & 0.53 & 0.18 & & 0.27 & 0.06 & 0.12 & .028 & 0.2 & 26 & 0.16 & 1 & & & & & & & & & & & & \\
\hline 11 Insuriance and financial services (\% of commercial service impotts) & 0.92 & 0.85 & 0.12 & 0.2 & & 0.54 & .041 & .0 .09 & 0.77 & 0.8 & 85 & 0.80 & .048 & 1 & & & & & & & & & & & \\
\hline 12 Ful imports (\% of merchandise impots) & 0.89 & 0.88 & 0.05 & 0.1 & & 0.45 & .0 .57 & 0.15 & 0.77 & 0.8 & 88 & 0.84 & .058 & 0.91 & 1 & & & & & & & & & & \\
\hline 13 Food imports (\% of merchandise impots) & .0 .78 & .0 .7 & 0.01 & .033 & & 0.44 & 0.62 & 0.01 & .058 & .07 & 77 & 0.66 & 0.21 & .081 & .0 .76 & 1 & & & & & & & & & \\
\hline 14 Agriculdural aw material imports (\% of merchandise imports) & .0 .73 & 0.71 & 0.13 & .039 & & .050 & 0.51 & 0.12 & .034 & $\cdot 0.7$ & 76 . & 0.68 & 0.22 & 0.69 & .0 .71 & 0.73 & 1 & & & & & & & & \\
\hline 15. Taiff rate, most favored nation, weighted mean, primary products (\%) & .047 & .0 .62 & 0.16 & 0.24 & & .039 & 0.38 & 0.4 & .058 & .0 .5 & 57 & .059 & 0.25 & .0 .50 & .056 & 0.38 & 0.33 & 1 & & & & & & & \\
\hline 16 Taiff frate, applied, weighted mean, primary products $(\%)$ & .0 .64 & .0 .78 & 0.29 & .033 & & .057 & 0.49 & 0.05 & .0 .69 & -0.7 & 73 & .0 .77 & 0.21 & .0 .64 & .0 .69 & 0.51 & 0.77 & 0.95 & 1 & & & & & & \\
\hline 17. Share of tariffll lines with specific rates, primary products $(\%)$ & .0 .68 & .051 & 0.11 & .018 & & .0 .15 & 0.5 & 0.02 & .031 & .05 & 56 & 0.46 & 0.46 & .0 .60 & .0 .59 & O.4 & 0.56 & .0 .04 & 0.12 & 1 & & & & & \\
\hline 18 Taifff rate, applied, simple mean, all products (\%) & .089 & .095 & 0.34 & .033 & & .073 & 0.59 & 0.05 & .0 .79 & .99 & 93 & .094 & 0.24 & .084 & .0 .86 & 0.74 & 0.70 & 0.71 & 0.86 & 0.5 & 1 & & & & \\
\hline 19. International tounism, number of arrivals & 0.89 & 0.92 & 0.12 & 0.26 & & 0.59 & .0 .58 & -0.07 & 0.77 & 0.9 & 91 & 0.84 & .031 & 0.88 & 0.87 & .080 & .070 & .052 & .067 & .0 .58 & 0.88 & 1 & & & \\
\hline 2a Trade (\% of GDP) & .0 .99 & 0.03 & 0.000 & 0.06 & & .0 .29 & .036 & .0 .22 & 0.12 & 0.0 & 02 & .0 .14 & $\cdot .13$ & 0.01 & 0.05 & .0 .22 & 0.10 & .030 & .0 .20 & 0.13 & 0.05 & 0.20 & 1 & & \\
\hline 21 Taxes on expotts (\% of tax revernue) & .0 .63 & 0.75 & 0.3 & 0.39 & & 0.72 & .048 & 0.05 & 0.62 & 0.7 & 71 & 0.72 & 0.23 & 0.60 & 0.50 & .0 .60 & .0 .51 & .034 & .0 .52 & -0.28 & 0.69 & 0.81 & 0.11 & 1 & \\
\hline 22 Trade in services (\% of GDP) & .0 .53 & .0 .4 & 0.00 & .006 & & 0.600 & -0.02 & 0.10 & .036 & 0.4 & 42. & .058 & .0 .05 & .0 .46 & 0.36 & 0.18 & 0.36 & 0.02 & 0.18 & 0.32 & 0.39 & .0 .26 & 0.83 & .0 .31 & \\
\hline
\end{tabular}

\section{Source: Author's calculations using Ms.Excel software.}

In this section, each indicator that had a correlation coefficient with the TFP that is greater than or equal to -0.5 or +0.5 was selected for running a multiple regression between agricultural TFP as a dependent variable and all the highly correlated indicators as explanatory variables. However, this second step was executed differently due to data availability of the mentioned indicators. There were divided into 3 groups according to data availability; 54 years (1961-2014), 44 years (1971-2014), and 25 years (1990-2014). The stationarity of each indicator in each group of data was tested using Augmented Dickey Fuller (ADF) test Eviews software Tables $14,15,16$. 


\section{Table 14.summary of the highly correlated indicators, and their stationarity} level.(1961-2014).

\begin{tabular}{|c|c|c|c|c|}
\hline $\begin{array}{c}\text { Indicators according to } \\
\text { data availability (54 years) }\end{array}$ & $\begin{array}{l}\text { Indicator } \\
\text { abbrev. }\end{array}$ & $\begin{array}{c}\text { Correlation } \\
\text { with TFP }\end{array}$ & $\begin{array}{c}\text { Stationarity } \\
\text { Level* }\end{array}$ & $\begin{array}{c}\text { form iserted into } \\
\text { multiple regression }\end{array}$ \\
\hline \multicolumn{5}{|l|}{ TFP** } \\
\hline $\begin{array}{l}\text { Technical cooperation } \\
\text { grants (BoP, current US\$) }\end{array}$ & TCG & -0.89 & I(1) Random walk & D(TCG) \\
\hline $\begin{array}{l}\text { Mortality rate, under-5 } \\
\text { (per } 1,000 \text { live births) }\end{array}$ & MR & -0.95 & $\begin{array}{l}\text { Random walk } \\
\text { with trend }\end{array}$ & MR_Resids \\
\hline $\begin{array}{l}\text { Gross domestic savings } \\
\text { (current LCU) }\end{array}$ & GDS & 0.92 & $\begin{array}{l}\text { Random walk } \\
\text { with trend }\end{array}$ & GDS_Resids \\
\hline $\begin{array}{l}\text { Gross capital formation } \\
\text { (current LCU) }\end{array}$ & GCF & 0.93 & $\begin{array}{l}\text { Random walk } \\
\text { with trend }\end{array}$ & GCF_Resids \\
\hline $\begin{array}{l}\text { Gross national expenditure } \\
\text { (current LCU) }\end{array}$ & GNE & 0.89 & $\begin{array}{l}\text { Random walk } \\
\text { with trend }\end{array}$ & GNE_Resids \\
\hline $\begin{array}{l}\text { Official exchange rate } \\
\text { (LCU per US\$, period average) }\end{array}$ & OER & 0.90 & $\begin{array}{l}\text { Random walk } \\
\text { with trend }\end{array}$ & OER_Resids \\
\hline Merchandise exports(current US\$) & MEX & 0.93 & $\mathbf{I}(\mathbf{0})$ & MEX \\
\hline $\begin{array}{l}\text { Food exports } \\
\text { (\% of merchandise exports) }\end{array}$ & FOEX & -0.61 & $\begin{array}{l}\text { I(1) Random } \\
\text { walk }\end{array}$ & FOEX.Resids \\
\hline $\begin{array}{l}\text { Agricultural raw materials exports } \\
\text { (\% of merchandise exports) }\end{array}$ & ARMEX & -0.53 & $\begin{array}{l}\text { I(1) Random walk } \\
\text { with trend }\end{array}$ & $\begin{array}{c}\text { ARMEX } \\
\text { Resids }\end{array}$ \\
\hline $\begin{array}{l}\text { Merchandise imports } \\
\text { (current US\$) }\end{array}$ & MIM & 0.90 & $\begin{array}{l}\text { I(1) Random walk } \\
\text { with trend }\end{array}$ & MIM_Resids \\
\hline $\begin{array}{l}\text { Fuel imports } \\
\text { (\% of merchandise imports) }\end{array}$ & FUIM & 0.89 & $\begin{array}{l}\text { I(1) Random walk } \\
\text { with trend }\end{array}$ & FUIM_Resids \\
\hline $\begin{array}{l}\text { Food imports } \\
\text { (\% of merchandise imports) }\end{array}$ & FOIM & -0.78 & $\mathbf{I}(\mathbf{0})$ & FOIM \\
\hline $\begin{array}{l}\text { Agricultural raw materials imports } \\
\text { (\% of merchandise imports) }\end{array}$ & ARMIM & -0.73 & $\mathbf{I}(\mathbf{0})$ & ARMIM \\
\hline
\end{tabular}

* Stationarity was tested according to Augmented Dicky-Fuller test in Eviews ** TFP was estimated for 54 years (1961-2014)

Source: Author's calculations using eviews software.

Table 15.summary of the highly correlated indicators, and their stationarity level.(1971-2014).

\begin{tabular}{|c|c|c|c|c|}
\hline $\begin{array}{l}\text { Indicators according to data } \\
\text { availability (44 years) }\end{array}$ & $\begin{array}{l}\text { Indicator } \\
\text { abbrev. }\end{array}$ & $\begin{array}{c}\text { Correlation } \\
\text { with TFP }\end{array}$ & $\begin{array}{c}\text { Stationarity } \\
\text { Level* }\end{array}$ & $\begin{array}{c}\text { form iserted into } \\
\text { multiple regression }\end{array}$ \\
\hline TFP** & & & & \\
\hline $\begin{array}{l}\text { Net ODA received per } \\
\text { capita (current US\$) }\end{array}$ & NODA & 0.51 & $\begin{array}{l}\text { I(1) Random } \\
\text { walk }\end{array}$ & D_NODA \\
\hline $\begin{array}{l}\text { Employment in agriculture } \\
\text { (\% of total employment) }\end{array}$ & EIA-T & -0.69 & $\mathbf{I}(\mathbf{0})$ & EIA-T \\
\hline $\begin{array}{l}\text { Employment in agriculture, male } \\
\text { (\% of male employment) }\end{array}$ & EIA-M & -0.83 & $\begin{array}{l}\text { Random walk } \\
\text { with trend }\end{array}$ & EIA-M_Resids \\
\hline $\begin{array}{l}\text { Energy use } \\
\text { (kg of oil equivalent per capita) }\end{array}$ & $\mathbf{E} \mathbf{U}$ & 0.93 & $\begin{array}{l}\text { Random walk } \\
\text { with trend }\end{array}$ & EU_Resids \\
\hline $\begin{array}{l}\text { Electric power consumption } \\
\text { (kWh per capita) }\end{array}$ & EPC & 0.98 & $\begin{array}{l}\text { Random walk } \\
\text { with trend }\end{array}$ & EPC_Resids \\
\hline $\begin{array}{l}\text { Electricity production } \\
\text { from oil sources (\% of total) }\end{array}$ & EPFOS & -0.68 & $\begin{array}{l}\text { Random walk } \\
\text { with trend }\end{array}$ & EPFOS_Resids \\
\hline $\begin{array}{l}\text { Imports of goods and } \\
\text { services (BoP, current US\$) }\end{array}$ & IMGS & 0.94 & $\begin{array}{l}\text { Random walk } \\
\text { with trend }\end{array}$ & IMGS_Resids \\
\hline $\begin{array}{l}\text { Government expenditure on } \\
\text { education, total ( } \% \text { of GDP) }\end{array}$ & GEOE-T & -0.66 & $\mathbf{I}(\mathbf{0})$ & GEOE-T \\
\hline $\begin{array}{l}\text { Fossil fuel energy consumption } \\
\text { (\% of total) }\end{array}$ & FFEC-T & -0.93 & $\begin{array}{c}\text { Random walk } \\
\text { with trend }\end{array}$ & FFEC-T_Resids \\
\hline Lending interest rate $(\%)$ & LIR & -0.84 & $\begin{array}{l}\text { T(1) Random walk } \\
\text { with drift }\end{array}$ & D(LIR) \\
\hline Air transport, freight (million ton-km) & ATF & 0.75 & $\mathbf{I}(\mathbf{0})$ & ATF \\
\hline $\begin{array}{l}\text { Net lending (+)/ net borrowing } \\
(-)(\% \text { of GDP })\end{array}$ & NL/NB & -0.84 & I(0) & NL/NB \\
\hline $\begin{array}{l}\text { Commercial service imports } \\
\text { (current US\$) }\end{array}$ & CSI & 0.95 & $\begin{array}{l}\text { Random walk } \\
\text { with trend }\end{array}$ & CSI_Resids \\
\hline $\begin{array}{l}\text { Insurance and financial services } \\
\text { (\% of commercial service imports) }\end{array}$ & IFS & 0.92 & $\begin{array}{l}\text { Random walk } \\
\text { with trend }\end{array}$ & IFS_Resids \\
\hline
\end{tabular}

(\% of commercial service imports) with trend

$* *$ TFP was estimated for 54 years, however due to the data availability of the indicators, it was altered to each group. In this table, it was altered to be 44 years.

Source: Author's calculations using eviews software. 


\section{Table 16.summary of the highly correlated indicators, and their stationarity} level.(1990-2014).

\begin{tabular}{|c|c|c|c|c|}
\hline $\begin{array}{c}\text { Indicators according to } \\
\text { data availability ( } 25 \text { years) }\end{array}$ & $\begin{array}{c}\text { Indicator } \\
\text { abbrev. }\end{array}$ & $\begin{array}{l}\text { Correlation } \\
\text { with TFP }\end{array}$ & $\begin{array}{c}\text { Stationarity } \\
\text { Level }\end{array}$ & $\begin{array}{l}\text { form inserted into } \\
\text { multiple regression }\end{array}$ \\
\hline TFP** & & & & \\
\hline Rural population & $\mathbf{R P}$ & 0.98 & Random walk with trend & RP_Resids \\
\hline $\begin{array}{l}\text { Access to electricity, rural } \\
\text { (\% of rural population) }\end{array}$ & ATE-R & 0.95 & Random walk with trend & ATE-R_Resids \\
\hline $\begin{array}{l}\text { Permanent cropland } \\
\text { (\% of land area) }\end{array}$ & PC-T & 0.89 & Random walk with trend & PC-T_Resids \\
\hline $\begin{array}{l}\text { Land under cereal } \\
\text { production (hectares) }\end{array}$ & LUCP & 0.96 & Random walk with trend & LUCP_Resids \\
\hline $\begin{array}{l}\text { Renewable energy consumption } \\
\text { (\% of total final energy consumption) }\end{array}$ & REC & -0.94 & Random walk with trend & REC_Resids \\
\hline $\begin{array}{l}\text { Electricity production from renewable } \\
\text { sources, excluding hydroelectric (\% of total) }\end{array}$ & EPFRS/T & 0.92 & Random walk with trend & EPFRS/T_Resids \\
\hline $\begin{array}{l}\text { Electricity production from renewable } \\
\text { sources, excluding hydroelectric (kWh) }\end{array}$ & EPFRS & 0.90 & Random walk with trend & EPFRS_Resids \\
\hline Access to electricity (\% of population) & ATE & 0.95 & Random walk with trend & ATE Resids \\
\hline $\begin{array}{l}\text { Price level ratio of PPP conversion } \\
\text { factor (GDP) to market exchange rate }\end{array}$ & PLRppp & 0.65 & Random walk with trend & PLRppp_Resids \\
\hline Labor force, total & LF-T & 0.98 & Random walk with trend & LF-T_Resids \\
\hline $\begin{array}{l}\text { Improved water source } \\
\text { (\% of population with access) }\end{array}$ & IWS & 0.99 & Random walk with trend & IWS_Resids \\
\hline $\begin{array}{l}\text { Improved water source, rural } \\
\text { (\% of rural population with access) }\end{array}$ & IWSR & 0.98 & $\mathbf{I}(\mathbf{0})$ & IWSR \\
\hline Rail lines (total route-km) & $\mathbf{R L}$ & 0.86 & $\mathbf{I}(\mathbf{0})$ & $\mathbf{R L}$ \\
\hline Health expenditure, total (\% of GDP) & HE-T & 0.71 & Random walk with trend & HE-T_Resids \\
\hline Health expenditure, public (\% of GDP) & HE-P & 0.56 & Random walk with trend & HE-P Resids \\
\hline $\begin{array}{l}\text { Health expenditure, public } \\
\text { (\% of total health expenditure) }\end{array}$ & HEPT & -0.74 & Random walk with trend & HEPT_Resids \\
\hline Health expenditure, private (\% of GDP) & HE-Pr & $\mathbf{0 . 7 4}$ & $\mathbf{I ( 0 )}$ & HE-Pr \\
\hline Health expenditure per capita (current US\$) & HEPC & 0.90 & Random walk with trend & HEPC Resids \\
\hline $\begin{array}{l}\text { Depth of the food deficit } \\
\text { (kilocalories per person per day) }\end{array}$ & DFD & -0.57 & Random walk with trend & DFD_Resids \\
\hline Subsidies and other transfers (current LCU) & SOT & 0.88 & $\mathbf{I}(\mathbf{0})$ & SOT \\
\hline $\begin{array}{l}\text { Research and development } \\
\text { expenditure (\% of GDP) }\end{array}$ & RD-T & 0.78 & Random walk with trend & RD-T_Resids \\
\hline $\begin{array}{l}\text { Tariff rate, applied, simple } \\
\text { mean, all products }(\%)\end{array}$ & TR & -0.89 & Random walk with trend & TR_Resids \\
\hline $\begin{array}{l}\text { Transport services } \\
\text { (\% of commercial service imports) }\end{array}$ & TS & 0.73 & Random walk with trend & TS_Resids \\
\hline $\begin{array}{l}\text { Foreign direct investment, net } \\
\text { inflows (BoP, current US\$) }\end{array}$ & FDI-I & 0.60 & Random walk with trend & FDI-I_Resids \\
\hline $\begin{array}{l}\text { Foreign direct investment, } \\
\text { net outflows (BoP, current US\$) }\end{array}$ & FDI-O & 0.56 & Random walk with trend & FDI-O_Resids \\
\hline Trade in services (\% of GDP) & TIS & -0.53 & Random walk with trend & TIS_Resids \\
\hline exports $(\%$ of tax & TOEX & -0.63 & Random walk with trend & TOEX_ Resids \\
\hline International tourism, number of arrivals & IT & 0.89 & Random walk with trend & IT_Resids \\
\hline
\end{tabular}

** TFP was estimated for 54 years, however due to the data availability of the indicators, it was altered to each group. In this table, it was altered to be 25 years to fit into the multiple regression.

I (1): it means that the indicator is itegrated of order 1 or at 1 st difference; in this case the indicator is either Random walk or Random walk with a drift, in both cases the first difference (due to the level of integration, as shown in the table) was taken to represent the stationary form of the indicators.

I(0): integrated at level Resids: it means that the indicator was regressed with time-as it has a trend component-, the residuals of this regression were considered the new stationary indicator.

Source: Author's calculations using eviews software.

\section{TFP Determinants}

This section is mainly running multiple regressions after selecting the indicators that had a correlation coefficient that is above +0.5 or -0.5 . then running a multiple regression between the TFP as a dependent variable and the development 
indicators as explanatory variables. However, the possible multi sectoral determinants of agricultural TFP in Egypt are presented in three groups according to data availability and regardless of their sector; 54 years 1961-2014, 44 years 1971-2014, and 25 years 1990-2014 table 17.

Before running the multiple regressions, the stationarity level of all variables was tested using Augmented Dikkey Fuller test, then inserted into the regression analysis in stationary form as shown in tables 14,15 and 16 respectively. After the regression, the serial correlation was tested using Durbin Watson and BreuschGodfrey Serial Correlation LM Test. In addition to Heteroscedasticity tested using Breusch-Pagan-Godfrey test.

Table 17: TFP determinants in the Egyptian agricultural sector (1961-2014)

\begin{tabular}{|l|l|l|l|}
\hline $\begin{array}{l}\text { Dependent Variable ( LN TFP) } \\
\text { Parameters }\end{array}$ & Coefficient & T-statistic & Prob. \\
\hline OER & 0.971047 & 2.876041 & 0.0097 \\
\hline GDS & $2.87 \mathrm{E}-11$ & 4.820010 & 0.0001 \\
\hline ARMEX & -0.031255 & -0.793356 & 0.4374 \\
\hline Constant & 1.761829 & 4.025130 & 0.0007 \\
\hline \multicolumn{2}{|l|}{} & & \\
\hline R-squared & & & 0.594983 \\
\hline F-statistic (prob.) & & 0.000537 \\
\hline Durbin Watson & & 1.561265 \\
\hline Breusch-Godfrey Serial Correlation LM Test (p-value) & & 0.9946 \\
\hline Heteroskedasticity Test: Breusch-Pagan-Godfrey (p-value) & & 0.1300 \\
\hline
\end{tabular}

TFP $=\mathrm{f}($ GDS,OER, ARMEX)

Where:

TFP = Total Factor Productivity in the Egyptian agricultural sector;

GDS $\quad(+)=$ Gross domestic savings (current LCU)

OER $(+)=$ Official exchange rate (LCU per US\$, period average)

ARMEX (-) = Agricultural raw materials exports (\% of merchandise exports)

Source: Author's calculations using Eviews software.

Fig.1. Summary of Regression results of the TFP determinants 1961-2014

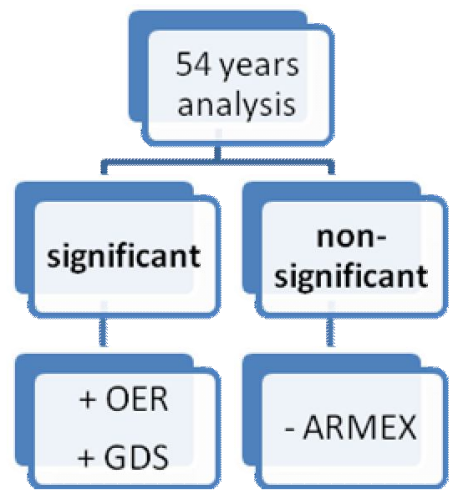

Source: Author's elaboration.

The log-linear form of the above equation shown in table 17 and summarized in figure 1, allows for estimating coefficients that can be directly interpreted as elasticities. The estimations shown in table 17 indicate that: (i) the estimation output 
have no serial correlation according to the Durbin Watson Value 1.56 and this is confirmed by the non-significant(more than .05) p-value of Breusch-Godfrey Serial Correlation LM Test 0.994. On the other hand there is no heteroscedasticity according to the non-significant p-value of Breusch-Pagan-Godfrey test 0.13. (ii) The relationship among the TFP in the Egyptian agricultural sector and each of Gross Domestic Product (GDS), Official Exchange Rate (OER) is positive and statistically significant. But, the relationship among the TFP in the Egyptian agricultural sector and ARMEX is negative and statistically non-significant.

As for the Official exchange rate (OER), from a general perspective in a market-based economy; household, producer, and government choices about resource allocation are influenced by relative prices, including the real exchange rate, real wages, real interest rates, and other prices in the economy. Relative prices also largely reflect these agents' choices. Thus relative prices convey vital information about the interaction of economic agents in an economy and with the rest of the world.

On the other hand the depreciating, however relatively stable exchange rate causes the agricultural exports to be cheaper therefore more competitive in the global market, thus more demand for exports. This creates opportunities for processing leading to more value added, increasing exporter's income creating additional demand. So the devaluation of the OER could cause a boost to the economic growth. Habib, M.M et. al., (2016) found that currency depreciation raises annual real GDP growth only for the developing countries. In addition, Gluzmannet. al. (2012) found that the undervaluation leads to greater domestic savings and therefore investment in the developing countries.

- Agricultural raw materials exports (ARMEX), had a negative effect on TFP comes as no surprise. The specialization of some countries in the export of raw materials and lightly processed goods is an important cause of their underdevelopment, Delacroix, J. (1977). It is proved that raw material exports is a fast but rather shorthanded way of making profit for any exporter. Also, it is a strong deterrent of growth regardless of the sector. Egypt's government supports export-orientedactivities that increase the value added, but not the raw materials. The government supports funds that aim at developing production practices for improving export capabilities. Similarly, the Export Development Bank of Egypt provides short and medium term loans to finance inputs for export industries. Moreover, the processed agricultural products are among the beneficiaries of such credits.

Gross Domestic Savings (GDS), was proved to be positively associated with productivity growth in poor countries (Aghion, P. 2009) Domestic saving is more critical for adopting new technologies in developing rather than developed economies.

The linear form of the above equation, the estimations shown in table 18 and summarized in figure 2 indicates that: (i) the estimation output has no serial correlation according to theDurbin Watson Value 2.01 and this is confirmed by the non-significant(more than .05) p-value of Breusch-Godfrey Serial Correlation LM Test 1.0. On the other hand there is no heteroscedasticity according to the non- 
significant $p$-value of Breusch-Pagan-Godfrey test 0.15. (ii) The relationship among the TFP in the Egyptian agricultural sector and each of Net Official Development Assistance (NODA), Electric Power Consumption (EPC), Imports of goods and services (IMGS) is positive and statistically significant. But, the relationship among the TFP in the Egyptian agricultural sector andFossil Fuel Energy Consumption (FFEC) is negative and statistically non-significant.

Table 18: TFP determinants in the Egyptian agricultural sector (1971-2014)

\begin{tabular}{|l|l|l|l|}
\hline $\begin{array}{l}\text { Dependent Variable ( LN TFP) } \\
\text { Parameters }\end{array}$ & Coefficient & T-ratio & P-value \\
\hline IMGS & $1.18 \mathrm{E}-10$ & 4.921088 & 0.0004 \\
\hline EPC & 0.008076 & 4.927391 & 0.0003 \\
\hline NODA & 0.025501 & 3.960465 & 0.0019 \\
\hline FFEC & -0.216012 & -1.401194 & 0.1865 \\
\hline Constant & 1.445861 & 7.415390 & 0.0000 \\
\hline \multicolumn{2}{|l}{} & & \\
\hline R-squared & & & 0.878231 \\
\hline F-statistic (prob.) & & & 0.000020 \\
\hline Durbin Watson & & 2.016678 \\
\hline Breusch-Godfrey Serial Correlation LM Test (p-value) & & 1.0000 \\
\hline Heteroskedasticity Test: Breusch-Pagan-Godfrey (p-value) & & 0.1532 \\
\hline
\end{tabular}

TFP = f (NODA,EPC, IMGS,FFEC $)$

Where:

TFP = Total Factor Productivity in the Egyptian agricultural sector;

NODA $(+)=$ Net ODA received per capita (current US\$)

EPC $(+)=$ Electric power consumption (kWh per capita)

IMGS $(+)=$ Imports of goods and services (BoP, current US\$)

FFEC (-) = Fossil fuel energy consumption (\% of total)

Source: Author's calculation using Eviews software.

Fig2. Summary of Regression results of the TFP determinants 1971-2014

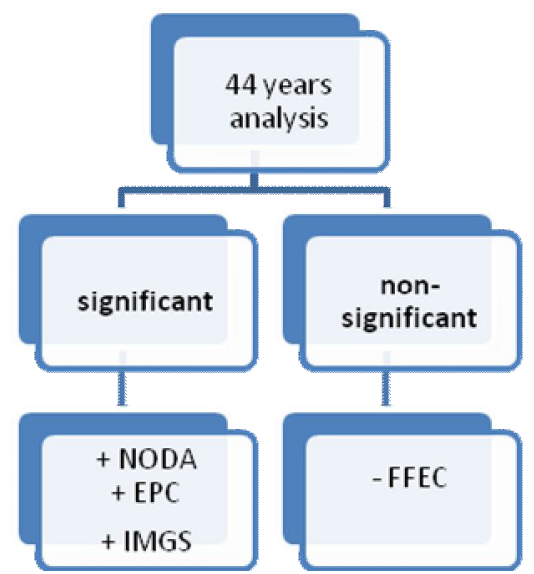

Source: Author's elaboration.

- As for Net Official Development Assistance (NODA), The Foreign aid can play an important and vital role in realizing the development goals and poverty reduction especially in the developing countries. The coordination of aid focuses on the procedures that lead to transfer the official development assistance to private institution or government recipient. Egypt still needs to develop its capacities, human resources and solve the difficulties in the resource management and investments. These difficulties have a negative effect on the country's potential for undertaking an accelerated process of development. Egypt needs to ensure efficient 
allocation of both internal and external resources to increase the level of development. The ministry of international cooperation in Egypt is mandate to achieve the optimum utilization of external assistance to improve the effectiveness of Egypt's development programs. Few studies have focused on evaluating the effect of aid on economic growth in some developing countries(Roodman, 2006).

The major donors to Egypt are the United States Agency for International Development (USAID), European Commission (EC), European member states (EU), Japan and the World Bank. The United States is the dominant donor for Egypt accounting for $50 \%$ of the total development assistance in 1990s. The United Nation agencies contributed $1.3 \%$ of all aid, while the USA and EU jointly contributed about $89 \%$ of the total aid to Egypt during this period. The international donors assisted Egypt in implementing the Economic Reform and Structural Adjustment Program (ERSAP) to promote the private sector and increase economic growth. Egypt received aid from the donors in the form of bilateral agreement $(51.84 \%)$ and multilateral agreement (48.19\%). NGOs represented by Ford Foundation that reached about $(0.0002 \%)$ and others $(0.0032 \%)$. Aou el nour, K., 2014.

The cumulative ratio indicates that USAID maintained its top rank as the first donor (66.77\%), Germany is the second (10.58\%), Italy (4.29\%), Abu Dhabi Fund (3.96\%) and Kuwait Fund (2.512\%). The other donors participated by ratio between $(1.052 \%$ up to $1.890 \%)$ such as Danish international Development Agency (DANIDA), Spain, Japan, Canadian International Development Agency (CIDA), Netherlands and Switzerland.

The analysis of ODA by geographic location indicate that the central government and lower Egypt governorates received the highest proportions of the total official development assistance disbursements. Therefore, the distribution of aid is biased for the urban governorates such as Alexandria (8.7\%) and Grand Cairo (7.66\%). In contrast, some governorates in Upper Egypt and out of valley such as Assyout, Suhag and north Sinai governorates received lower proportions of assistance, although they still need more water sanitation, health care and education services. Burnside and Dollar (1997) and Azamet al. (1999).

Agriculture sector: It ranked third among recipient sectors after energy and industry. It received $10.71 \%$ of the total value of disbursements by sectors. The allocation of aid funds to the agricultural research, industrial and export crops, post harvest and also agricultural development and livestock activities is still very low although, if increased, it can enhance and empower the agricultural sector in Egypt. Where the majority of agricultural producers are small farmers. Supporting this sector does not only mean boosting the Egyptian national economy, but will also raise agricultural production and increase agricultural exports. Moreover, supporting the agricultural sector will help carry out the national development policy for reducing poverty especially in the rural areas. Successful examples of aid effectiveness in Egypt:

\section{micro level (socio economic impacts of IEDS projects):}

started since the year 2000 for eight years, under the Italian and Egyptian cooperation development swap, with a total value of about L E 850 million. Such as 
West Noubaria Rural Development Project, employment generation and poverty alleviation. The farmers benefited from improved living standards and increased micro enterprises for women's. The program led to other projects such as establishment of databases in rural areas. Also, the sustainable rural development of Wadi El Rayan, New Land Settlements and Marketing Link Program.

\section{macro level (aid effectiveness on the national capacity development in Egypt)}

Economic policy initiative consortia (EPIC)1996: a four years period and was financed by the USAID as provided advice on economic policy reform to Egyptian policy makers and introduced technical support to improve the Egyptian performance in national development.

Education sector assistance: The government of Egypt has worked with the European Commission and the World Bank since 1996 on the framework of education reform.

UNDP program at the Institute of National Planning (INP): This program received financial and technical support from United Nations Development Program (UNDP), United Nations Population Fund (UNFPA), World Food Program (WFP) and the Social Fund for Development (SFD) in Egypt. The program aimed at providing an annual report on human development in Egypt

Banking institute: This institute was financed by different donors such as (European Commission (EC), United States Agency for International Development (USAID), United Kingdom (UK) and the Canadian International Development Agency (CIDA). The institute's objective was to enhance the capacity for development, expertise of Egyptian bankers and strengthen the banking leadership. What could be done in this regard is:

- Loans should be kept to its minimum values to limit indebtedness levels and not burden the country with heavy external debts. Better management and coordination of external assistance will increase the positive impact and efficiency of aid system. Raising technical and financial support will improve the performance of projects financed by donors. Also, maximizing the utilization of external financial resources will make positive impacts on the national development capacity of Egypt.

- Fragmented aid that comes in many small slices from a large number of donors creates high transaction costs and makes it difficult for partner countries effectively to manage their own development. Aid fragmentation also increases the risk of duplication and inefficient aid allocation among donors.

- Conducting development plans that are specific to each governorate especially those which receive lower proportions of assistance.

- Electric Power Consumption (EPC), In developing economies growth in energy use is closely related to growth in the modern sectors - industry, motorized transport, and urban areas - but energy use also reflects climatic, geographic, and economic factors (such as the relative price of energy). Energy use has been growing rapidly in low- and middle-income economies. An economy's production and consumption of electricity are basic indicators of its size and level of development. Although a few countries export electric power, most production is for domestic consumption. Expanding the supply of electricity to meet the 
growing demand of increasingly urbanized and industrialized economies without incurring unacceptable social, economic, and environmental costs is one of the great challenges facing developing countries. Energy use also reflects climatic, geographic, and economic factors (such as the relative price of energy). Governments in many countries are increasingly aware of the urgent need to make better use of the world's energy resources. Improved energy efficiency is often the most economic and readily available means of improving energy security. Comin and Hobijn (2004), proved that production of electricity have a positive effect on the degree of adoption of the current technology. Besides, Aschauer (1989) highlighted that physical infrastructure (for example, roads, water and sewage systems, and electricity supply) improvements are correlated with productivity. However, Hulten (1996) stressed that those countries that use infrastructure inefficiently pay a growth penalty in the form of a much smaller benefit from new infrastructure investment. The derived conclusion is that infrastructure is not only very important for productivity growth but even triggers it. However, it is important to closely monitor its management and financing.

- Imports of goods and services (IMGS);in an open-trade-regime, like Egypt, there is a better access to imported intermediate inputs of higher quality with a broad variety; this definitely enhances productivity. Egypt's main imports are machinery equipment, foodstuff, chemicals, and fuels. Each aspect of these imports affects the agricultural inputs and outputs, therefore the agricultural TFP. Keller and Yeaple (2003), established a positive link between imports and productivity growth. Also Coe and Helpman, (1995) highlighted the possibility that international $R \& D$ are driven by imports, most importantly machinery. Griffith, Redding and Van Reenen (2000) proved that Trade with a country on the world technology frontier showed a slight positive effect on TFP growth. Mayer (2001) argued that trade is a carrier of knowledge and focused on imports as a way of introducing foreign (relatively advanced) technology into domestic production, which in turn has a positive effect on TFP.

- Fossil Fuel Energy Consumption (FFEC), In developing economies growth in energy use is closely related to growth in the modern sectors - industry, motorized transport, and urban areas - but energy use also reflects climatic, geographic, and economic factors (such as the relative price of energy). Energy use has been growing rapidly in low- and middle-income economies. The current food system depends on non-renewable fossil fuel resources; its global status quo is scarce and expensive. This dependence is a threat to agriculture therefore the food supply. Egypt's fossil fuel consumption of total energy use reached almost 96\% in 2014. The consistent rises in fuel prices have their negative effect on agricultural income and agricultural growth. This effect is expected to deepen, especially after the government oil-subsidy- reduction-program. It will take decades for Egypt to substitute the non-environmentally friendly fossil fuel in agriculture or even to reduce its consumption. However, a lot could be done in that concern including a more efficient use of fossil fuel ; following the maintenance schedules of 
agricultural machinery, using energy-saving machinery, as well as fixing and constructing viable roads for efficient transport.

Table 19 : TFP determinants in the Egyptian agricultural sector (1990-2014)

\begin{tabular}{|l|l|l|l|}
\hline $\begin{array}{l}\text { Dependent Variable ( LN TFP) } \\
\text { Parameters }\end{array}$ & Coefficient & T-ratio & P-value \\
\hline FDI_I_RESIDS & $3.55 \mathrm{E}-10$ & 2.177021 & 0.0416 \\
\hline TOEX_RESIDS & -0.680107 & -1.166424 & 0.2572 \\
\hline RL & 0.002077 & 11.51679 & 0.0000 \\
\hline RD_RESIDS & 0.914530 & 1.878475 & 0.0750 \\
\hline Constant & -5.205000 & -5.725966 & 0.0000 \\
\hline & & & \\
\hline R-squared & & & 0.872238 \\
\hline F-statistic (prob.) & & & 0.000000 \\
\hline Durbin Watson & & & 2.593061 \\
\hline Breusch-Godfrey Serial Correlation LM Test (p-value) & & & 0.2362 \\
\hline Heteroskedasticity Test: Breusch-Pagan-Godfrey (p-value) & & 0.1023 \\
\hline
\end{tabular}

TFP = f (TOEX, RL, RD, FDII )

Where:

TFP = Total Factor Productivity in the Egyptian agricultural sector;

TOEX $(-)=$ Taxes on exports ( $\%$ of tax revenue)

$\mathbf{R L}(+)=$ Rail lines (total route-km)

RD $(+)=$ Research and development expenditure (\% of GDP)

FDII $\quad(+)=$ Foreign direct investment, net inflows (BoP, current US\$)

Source: Author's calculation using Eviews software.

Fig.3. Summary of Regression results of the TFP determinants 1990-2014

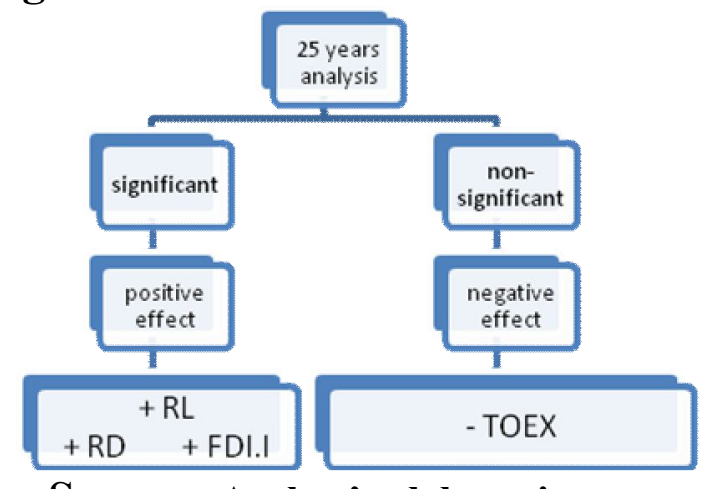

Source: Author's elaboration.

The linear form of the above equation, shown in table 19 and summarized in figure 3 the estimations shown in table 19 indicate that: (i) the estimation output have no serial correlation according to the Durbin Watson Value 2.5 and this is confirmed by the non-significant (more than .05) p-value of Breusch-Godfrey Serial Correlation LM Test 0.236 . On the other hand there is no heteroscedasticity according to the nonsignificant $p$-value of Breusch-Pagan-Godfrey test0.102. (ii) The relationship among the TFP in the Egyptian agricultural sector and each of RD, RL, FDII is positive and statistically significant. But, the relationship among the TFP in the Egyptian agricultural sector and of TOEX is negative and statistically non-significant.

Transport Services (TS), Ellis, S.D. and Hine.J.L.(2001) examined the relationship between accessibility, marketing and agricultural development; and found that transport plays a crucial role in identifying the link between accessibility 
and agricultural development. Ajiboye, A.O.andAfolayan, O.(2009), revealed that improved transportation will encourage farmers to work harder in the rural areas to increase production and products value added as well as the reduction of spoilage and wastage. Afterall increasing productivity and income.Reducing rural transport costs can raise farm-gate prices, increase farmers' incomes and help reduce the price of food in urban areas. It can also facilitate timely distribution of farm inputs (e.g. fertiliser, insecticide), increase agricultural yields and extend cultivated areas, and reduce post-harvest losses. Yet at present, rural transport systems in most developing countries, particularly in Africa, are still far from optimal.

Strategies for Improving Rural Transport and Agriculture: addressing inefficiencies and monopolistic practices of rural transport operators; improving efficiency of overall post-harvest storage and marketing operations; Improve load consolidation practices to reduce costs and increase bargaining power for farmers; Developing modern agricultural supply chains, particularly for high-value export crops and to meet demand of proliferating supermarkets; Increasing resilience to climate impacts, including through rural road improvements; and Establishing farmer's associations or cooperatives to lower the price of transport by arranging and purchasing farm inputs (such as fertilizer) in bulk

Research and Development (RD), despite the fact that all literature proved that R\&D is the main source of long term growth \{ Furman and Hayes(2004), Ulka(2004), Jones(2004), Chen and Dahlman(2004)\}; R\&D is often said to have two faces: the first is innovation, while the second is to facilitate the understanding and imitation of others' discoveries.Guellec and van Pottelsberghe de la Potterie (2001), displayed three sources of R\&D were considered,namely, domestic business research, public research (for example, by universities) andbusiness research undertaken by other countries. The first and third sources lead to newgoods and services, higher output quality and new production processes, while the secondone generates and increases basic and scientific knowledge. The results show that allthree sources of $\mathrm{R} \& \mathrm{D}$ are important for TFP growth, with foreign-sourced $\mathrm{R} \& \mathrm{D}$ having the largest effect. While public R\&D only produces scientific knowledge, and there is a very weak linkage between public reseachresults and the application process.Ahn (2001) argues that, in reality, it is not innovation input (in other words, R\&D investment) per se that counts for productivity, but the actual use of innovation output (in other words, use of advanced technology). Hasan (2002) pointed that investment in disembodied capital affects productivity positively only if it is of foreign origin; in house R\&D is never statistically significant. This all is to be in line with aninstitutional view, which suggests that countries with strong institutions achieve a higher output from investment in R\&D.

Foreign Direct Investment Inflows (FDI), Knowledgeis created by a small number of leader countries in technological terms. It could be imported through several channels. One important channel can be FDI, which theoretically brings knowledge into a country. R\&D activities in foreign countries, and thus contact with such countries, have been shown to spur growth domestically.FDI is believed to generate positive externalities in the form of knowledge spillovers to the domestic 
economy. However, negative externalities are also possible as barriers to accessing technology and competition may be raised. In the literature, the view adopted is often that positive externalities outweigh the negative ones and, for this reason, FDI is generally seen as a welcome addition to the domestic economy. In many cases, FDI is also encouraged (by governments and often also by international organizations) by offering grace periods for taxation purposes and different business support schemes. Hanafy, S.(2015) showed the sectoral distribution of FDI inflows to Egypt by origin in average (1972-2009) The fraction of Arab FDI that targets manufacturing is a bit smaller than non-Arab FDI, whereas a slightly larger share of Arab FDI targets the finance, tourism and agriculture sectors. Manufacturing sector accounted for $43 \%$, agriculture $4 \%$, aggregated services $53 \%$ ( finance $24 \%$ m tourism $11 \%$, construction $6 \%$, ICT $3 \%$, others $9 \%$ ).Agricultural FDI at the National Level, there was a significant increase of real FDI inflows to the agriculture sector in the second half of the last decade. The biggest share of FDI to agriculture targets land reclamation and cultivation (FAO, 2009).Despite the relatively small amount and fraction of FDI inflows that target the agriculturalsector, FDI on average contributed $18 \%$ of the sector's private investments since the 1970s the contribution has grown in the last two decades, reaching $24 \%$ of privateinvestments in the $2000 \mathrm{~s}$. This indicates that private investment in agriculture in Egypt isrelatively low, which, according to FAO (2009), is one of the sector's major problems.

As to the source of agricultural FDI, Arab countries accounted for two-thirds (67\%) onaverage however, agricultural FDI accounts for only 5\% of Arab FDI inflows to Egypt. Hanafy, S. (2015)

Agricultural FDI at the Governorate Level, agricultural FDI is highly concentrated in Cairo, which accumulated 44\% of the sector's FDI-stock. Despite the strong spatial concentration of agricultural FDI in general and in Cairo in particular, the agricultural sector still shows the second highest geographical dispersion of FDI after the manufacturing sector.Agricultural FDI is mostly concentrated in Cairo (44\%), Lower Egypt (22\%), Giza (13\%) and Upper Egypt (12\%), that is, along the Nile River, whereas a smaller amount of agriculture FDI stock targeted the Suez Canal governorates (5\%), the Frontier governorates (3\%), and Alexandria (2\%). Lower Egypt's governorate Sharkia actually ranks second after Cairo among the 27 Egyptian governorates in accumulating FDI stock (15\%) and Upper Egypt's Aswan ranks fourth $(10 \%)$. Although Giza (13\%) ranks third in agricultural FDI, its fraction in agricultural FDI is less than half its fraction in aggregate FDI stock. Similar to the manufacturing sector,but different from all service sub-sectors, all Egyptian governorates received some agricultural FDI flows during the last four decades. However, and again similar to manufacturing, 15 of the 27 governorates $(56 \%)$ accumulated less than $1 \%$ of agricultural FDI stock.

Detailed breakdown of economic activities in agriculture, reclamation and cultivation, stock breeding (livestock), poultry farming, fishing, slaughter houses

Taxes on Exports (TOEX), export taxes on agricultural products created a bias against agriculture in developing countries during the $1980 \mathrm{~s}$ Jensen, T., et. al.(2002).export taxes are the most commonly employed form of export restrictions 
on agricultural products, Estrades et al. (2017). They are applied either as a percentage of product value (an ad valorem tax) or as a fixed rate per physical unit of product (a specific tax)Kazeki(2006).According to FAO policy brief (October 2017) Countries usually apply the export restrictions in an attemptto contain the growth of domestic prices and ensure sufficient internal supplies, however these measures served as disincentives to farmers in exporting countries. In addition, evidence shows that export restrictions were not effective at preventing an increase in food prices, since the value share of the primary product (for example, wheat) in the final price of food (such as bread) is relatively low, ranging between 10 and 20 percent. Regions that apply export taxes would have an increase in production and exports if they removed export taxes. Estrades et al. (2017).

Egypt does not have any direct export subsidies. However, producers and exporters are supported through incentives such as tax concessions, and loans from the Export Development Bank of Egypt (EDBE). The EDBE was established in 1983, under Law 95; its main purpose was to encourage the development of Egyptian exports. It provides short- and medium-term loans to finance capital assets of export companies, and bank guarantees required for financing exports either directly to the exporter, or through other banks. The Bank also provides credit to finance imports primarily meant as inputs for export production, and acts as an insurer for exports against commercial and non-commercial risks. Around 200 projects were approved by the Bank in 1997. At the time of Egypt's Review in 1992, it was estimated that around $25 \%$ of Egypt's non-traditional exports were financed by the EDBE. To avoid the negative repercussions of quantitative export restrictions, it would be useful to have in place an improved, multilaterally agreed regulatory framework governing the use of these measures. According to ERS-USDA alternatives to a conventional export tax: (1) a consumption subsidy, (2) a production tax, and (3) a modification of a conventional export tax that allows additional exports after producers meet a domestic sales requirement.

\section{Conclusion and Recommendations}

The agricultural total factor productivity (TFP) growth provides society with opportunities to increase the welfare of people. It is, therefore, worth asking what determinants should policy makers focus on to enhance the performance of the agricultural TFP?. Having the calculated TFP as given from the previous work of Abdelrahman, N. (2018). The development indicators (determinants) considered here are grouped under 18 headings (sectors). They were used to figure out more explanatory variables the influence the agricultural TFP in Egypt:

agriculture and rural development, aid effectiveness, climate change, economy and growth, gender, public sector, private sector, poverty, infrastructure, science and technology, public health, social protection and labor, trade, urban development, environment, financial sector, external dept., and finally education.

By running a multiple regression between the TFP as a dependant variable and the development indicators as explanatory variables. However, the possible multi sectoral determinants of agricultural TFP in Egypt are presented in three groups 
according to data availability and regardless of their sector; 54 years 1961-2014, 44 years 1971-2014, and 25 years 1990-2014.

As for the 54-year-analysis, the relationship among the TFP in the Egyptian agricultural sector and each of GDS, OER is positive and statistically significant. But, the relationship among the TFP in the Egyptian agricultural sector and each of ARMEX is negative and statistically insignificant. As for the 44-year-analysis, the relationship among the TFP in the Egyptian agricultural sector and each of NODA, EPC, IMGS is positive and statistically significant. But, the relationship among the TFP in the Egyptian agricultural sector and FFEC is negative and statistically insignificant. As for the 25-year-analysis, the relationship among the TFP in the Egyptian agricultural sector and each of RD, RL, FDII is positive and statistically significant. But, the relationship among the TFP in the Egyptian agricultural sector and TOEX is negative and statistically insignificant.

- The depreciating, however relatively stable exchange rate causes the agricultural exports to be cheaper therefore more competitive in the global market, thus more demand for exports. This creates opportunities for processing leading to more value added, increasing exporter's income creating additional demand.

- Trade with a country on the world technology frontier showed a slight positive effect on TFP growth. Also, trade is a carrier of knowledge and focused on imports as a way of introducing foreign (relatively advanced) technology into domestic production, which in turn has a positive effect on TFP.

- Supporting export-oriented-activities that increase the value added, but not the raw materials. The government supports funds that aim at developing production practices for improving export capabilities.

- The agricultural sector ranked third among recipient sectors of net official development after energy and industry. The allocation of aid funds to the agricultural research, industrial and export crops, post-harvest and also agricultural development and livestock activities is still very low although, if increased, it can enhance and empower the agricultural sector in Egypt. Where the majority of agricultural producers are small farmers. Supporting this sector does not only mean boosting the Egyptian national economy, but will also raise agricultural production and increase agricultural exports. Moreover, supporting the agricultural sector will help carry out the national development policy for reducing poverty especially in the rural areas.

- The analysis of ODA by geographic location indicate that the central government and lower Egypt governorates received the highest proportions of the total official development assistance disbursements. Therefore, the distribution of aid is biased for the urban governorates such as Alexandria (8.7\%) and Grand Cairo (7.66\%). In contrast, some governorates in Upper Egypt and out of valley such as Assyout, Suhag and north Sinai governorates received lower proportions of assistance, although they still need more water sanitation, health care and education services. What could be done in this regard is:

- Loans should be kept to its minimum values to limit indebtedness levels and not burden the country with heavy external debts. Better management and 
coordination of external assistance will increase the positive impact and efficiency of aid system. Raising technical and financial support will improve the performance of projects financed by donors. Also, maximizing the utilization of external financial resources will make positive impacts on the national development capacity of Egypt.

- Fragmented aid that comes in many small slices from a large number of donors creates high transaction costs and makes it difficult for partner countries effectively to manage their own development. Aid fragmentation also increases the risk of duplication and inefficient aid allocation among donors.

- Conducting development plans that are specific to each governorate especially those which receive lower proportions of assistance.

- Infrastructure is not only very important for productivity growth but even triggers it. However, it is important to closely monitor its management and financing. Production of electricity has a positive effect on the degree of adoption of the current technology. Improved energy efficiency is often the most economic and readily available means of improving energy security.

- Agricultural labor could be characterized as casual or informal with low skill and productivity. On the other hand, the technological absorptive capacity of the country, an essential determinant of TFP growth, increases along with well-trained employment. A large share of agricultural labor is untrained which produces inefficiency in every aspect of the agricultural production that needs human labor. Institutions are weak, strengthening the role of agricultural institutions and giving high attention to agricultural labor training programs either the domestically funded or the internationally funded.

- Adopting more efficient use of fossil fuel ; following the maintenance schedules of agricultural machinery, using energy-saving machinery, as well as fixing and constructing viable roads for efficient transport; this idea could be transmitted to rural areas through media alongside with agricultural extension.

- Rural Transport services improving through : addressing inefficiencies and monopolistic practices of rural transport operators; improving efficiency of overall post-harvest storage and marketing operations; Improve load consolidation practices to reduce costs and increase bargaining power for farmers; Developing modern agricultural supply chains, particularly for high-value export crops and to meet demand of proliferating supermarkets; Increasing resilience to climate impacts, including through rural road improvements; and Establishing farmer's associations or cooperatives to lower the price of transport by arranging and purchasing farm inputs (such as fertilizer) in bulk

- Domestic Research and development was found insignificant; this is to be in line with an institutional view, which suggests that countries with strong institutions achieve a higher output from investment in R\&D.

- Adopting foreign and domestic policies that encourages FDI, as it is a strong growth stimulant and redirecting it to give more priority to the agri-labor training as well as the neglected governorates. 
- To avoid the negative repercussions of quantitative export restrictionssuch as export taxes, it would be useful to have in place an improved, multilaterally agreed regulatory framework governing the use of these measures. According to ERSUSDA alternatives to a conventional export tax: (1) a consumption subsidy, (2) a production tax, and (3) a modification of a conventional export tax that allows additional exports after producers meet a domestic sales requirement.

\section{Refrences}

- Ahn, S. (2001), "Firm Dynamics and Productivity Growth: A Review of Micro Evidence from OECD Countries", Economics Department Working Papers No. 297, Paris: OECD.

- Ajiboye, Araoye\& O, Afolayan. (2009). The impact of transportation on agricultural production in a developing country: a case of kolanut production in Nigeria. International Journal of Agricultural Economics and Rural Development. Vol. 2,.49-57.

- Alterman, J.B., 2006. Review of U.S. assistance programs to Egypt. Center for $\begin{array}{llll}\text { Strategic and International } & \text { Studies, }\end{array}$ http://csis.org/files/media/csis/congress/ts060621alterman.pdf.

- Aschauer, D.A. (1989), “Is Public Expenditure Productive?”,Journal of Monetary Economics, Vol. 23, pp. 177-200.

- Azam, F.P., S. Devarajan and S.A. O'Connell, 1999. Aid dependence reconsidered. World Bank, Washington, DC., USA., pp : 1-14. http://www.csae.ox.ac.uk/workingpapers/pdfs/9905text.pdf.

- Breusch, T. S. (1978). "Testing for Autocorrelation in Dynamic Linear Models". Australian Economic Papers.17: 334-355. doi: $\underline{10.1111 / j .1467-}$ 8454.1978.tb00635.x

- Burnside, C. and D. Dollar, 1997.Aid, policies and growth. Policy Research Paper No. 1777, The World Bank, Washington, DC., USA., pp: 1-64.

- Chen, D.H.C. and C.J. Dahlman (2004), "Knowledge and Development: A CrossSection Approach", Policy Research Working Paper, No. 3366, Washington, DC: World Bank.

- Coe, D.T: and E. Helpman (1995), "International R\&D Spillovers", Economic European Review, Vol. 39, pp. 859-87.

- Comin, D. and B. Hobijn (2004), "Cross-Country Technology Adoption: Making the Theories Face the Facts", Journal of Monetary Economics, Vol. 51, pp. 39-83.

- Delacroix, J. (1977). The Export of Raw Materials and Economic Growth: A CrossNational Study. American Sociological Review,42(5), 795-808. Retrieved from http://www.jstor.org/stable/2094867

- Estrades, C, M Flores and G Lezama (2017), "The role of export restrictions in agricultural trade," IATRC, Commissioned Paper 20.

- Furman, J.L. and R. Hayes (2004), "Catching Up or Standing Still? National Innovative Productivity Among "Follower" Countries, 1978-1999", Research Policy, Vol. 33, pp. 1329-54. 
- Glüzmann, P. A., Levy-Yeyati, E. and F. Sturzenegger (2012): "Exchange rate undervaluation and economic growth.", Economics Letters, 117, 3, 666-672.

- Griffith, R., Redding, S. and J. Van Reenen (2000), "Mapping the Two Faces of R\&D: Productivity Growth in a Panel of OECD Industries", CEPR Discussion Paper no. 2457, London: CEPR.

- Guellec, D. and B. van Pottelsberghe de la Potterie (2001), "R\&D and Productivity Growth: Panel Data Analysis of 16 OECD Countries", OECD Economic Studies No. 33, pp. 103-26.

- Hanafy, Shima'a (2015) : Patterns of foreign direct investment in Egypt: Descriptive insights from a novel panel dataset at the governorate level, Joint Discussion Paper Series in Economics, No. 12-2015, Univ., Dep. of Business Administration \& Economics, Marburg.

- Hasan, R. (2002), "The Impact of Imported and Domestic Technologies on the Productivity of Firms: Panel Data Evidence from Indian Manufacturing Firms", Journal of Development Economics, Vol. 69, pp. 23-49.

- Hulten, C.R. (1996), "Infrastructure Capital and Economic Growth: How Well You Use It May Be More Important Than How Much You Have", NBER Working Paper No. 5847, Cambridge, MA: NBER.

- Isaksson, A. (2001), "The Importance of Human Capital for the Trade-Growth Link", UNIDO Working Paper No. 2, Vienna: UNIDO.

- Jayson Beckman, Carmen Estrades, Manuel Flores, and Angel Aguiar, The Impacts of Export Taxes on Agricultural Trade.( 2018) NBER Working Paper No. 24894. JEL No. F1,F13,Q17

- Jensen Tarp, Henning, Sherman Robinson, and Finn Tarp.2002. General Equilibrium Measures of Agricultural Policy Bias in Fifteen Developing Countries. International Food Policy Research Institute. International Trade Journal 16:105128.

- Jones, C.I. (1995), "R\&D-Based Models of Economic Growth", Journal of Political Economy, Vol. 103(4), pp. 759-84.

- Kazeki, Jan. Export Duties. 2006. OECD Trade Policy Studies Looking Beyond Tariffs The Role of Non Tariff Barriers in World Trade 2006, no. 1, OECD. http://www.oecd.org.

- Keller, W. and S.R. Yeaple (2003), "Multinational Enterprises, International Trade, and Productivity Growth: Firm-Level Evidence from the United States", NBER WorkingPaper No. 9504, Cambridge, MA: NBER.

- KhaledAbou El Nour , 2014. An Analytical Study of the Egyptian System of Aid Coordination and Management.The International Journal of Applied Economics and Finance, 8: 1-16. URL:https://scialert.net/abstract/?doi=ijaef.2014.1.16

- Kheir El Din, H. 2000. Egypt's exports under liberalization: Performance, prospects and constraints (1980-1998). In H. Nassar\& A. Aziz, eds. Egyptian exports and challenges of the 21st century. Cairo, Center for Economic and Financial Research and Studies, Cairo University. 
- Laborde, D, C Estrades and A Bouët (2013), "A global assessment of the economic effects of export taxes," The World Economy 36(10): 1333-1354.

- Lehmijoki, Ulla \&Palokangas, Tapio. (2011). The Long-Run Effects of Mortality Decline in Developing Countries. ForschungsinstitutzurZukunft der Arbeit Institute for the Study of Labor, Discussion Paper No. 5422 January 2011, IZA P.O. Box 724053072 Bonn, Germany.

- Maurizio Michael Habib, ElitzaMileva, and LivioStracca (2016): "The real exchange rate and economic growth: revisiting the case using external instruments, "Working Paper Series 1921, European Central Bank.

- Mayer, J. (2001), "Technology Diffusion, Human Capital and Economic Growth in Developing Countries", Discussion Papers, No. 154, Geneva: United Nations Conference on Trade and Development.

- Mohamed Masry, 2015, Does Foreign Direct Investment (FDI) Really Matter in Developing Countries? The Case of Egypt.Research in World Economy, Vol. 6, No. 4; 2015.ISSN 1923-3981 E-ISSN 1923-399X

- Department, Faculty of Business Administration, Arab Academy for Science and Technology (AAST), Miami, Alexandria, Egypt. URL:

http://dx.doi.org/10.5430/rwe.v6n4p64.

- Naglaa, A.Abdelrahman., Gamal Siam, and Ali Ibrahim (2018), Evolution of Agricultural Total Factor Productivity in Egypt. Egyptian Association of Agricultural Economics., volume 28, no.2, june B 2018. ISSN 1110-6832.

- Philippe Aghion\& Diego Comin\& Peter Howitt\& Isabel Tecu, 2009. "When Does Domestic Saving Matter for Economic Growth?,"Harvard Business School Working Papers 09-080, Harvard Business School.

- Roodman, D., 2006. An index of donor performance. Working Paper No. 67, Center for Global Development, pp: 1-51. http://cgdev.org/files/3646 file_WP67nov.pdf.

- Ulku, H. (2004), "R\&D, Innovation, and Economic Growth: An Empirical Analysis", IMF Working Paper, WP/04/185, Washington, DC: International Monetary Fund.

- Zetter, R. and M.E. Hamza, 1998.Egypt: The state, foreign aid and community participation in urban shelter projects. Int. Plann. Stud., 3: 185-205.



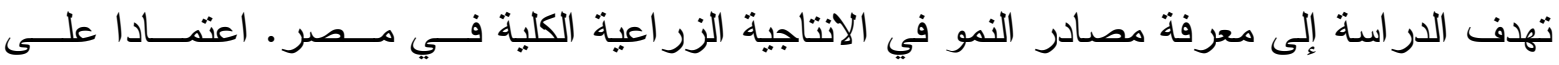

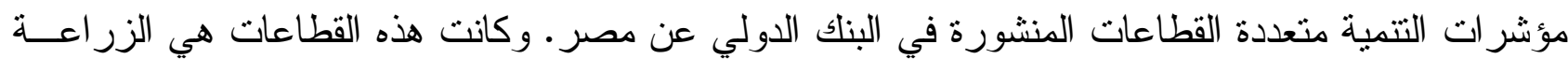

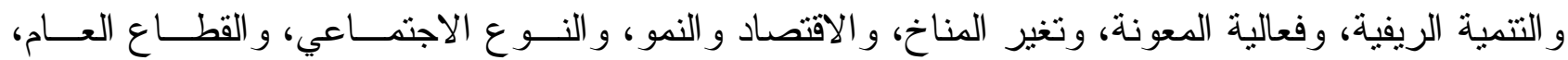

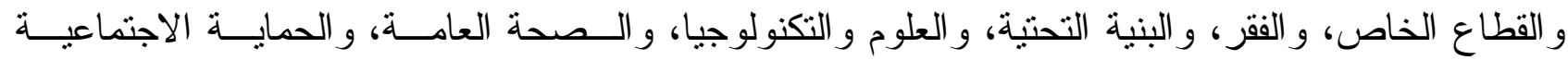

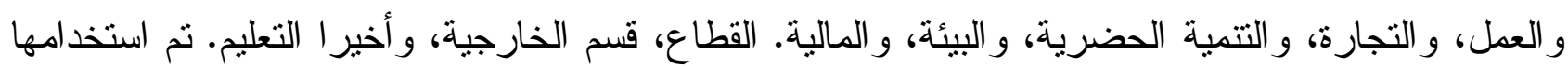
لمعرفة المزيد من المتغيرات التوضيحية التي تؤثر علي الإنتاجية الزر اعية الكلية (TFP) في مصر. 
وقد تحقق ذلك من خلا:

- توضيح العلاقة بين TFP ومجموعات المؤشر ات المذكورة أعلاه ؛ باستخدام مصفوفة معاملات الارتبــاط البسيط.

- اختيار المؤشرات المرتبطة بشكل كبير مع TFP و إجر اء تحليل متعمق (الانحدار المتعدد) للحصول علـىى

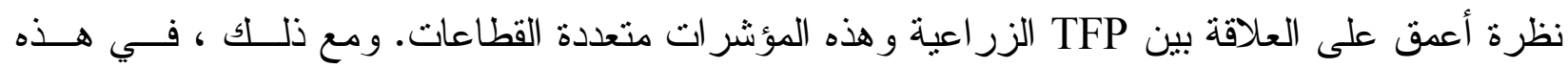

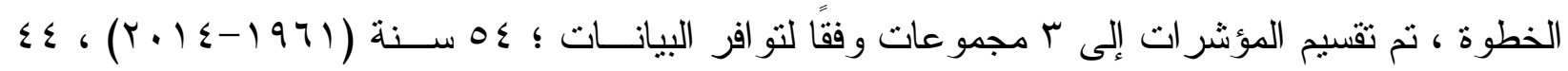
سنة (r) بالنسبة للتحليل( §ه سنة ) ، فإن العلاقة بين TFP في القطاع الزر اعي المصري وكل من الإدخــار المحلي الإجمالي، سعر الصرف الرسمي إيجابية و إحصائية ذات دلالة إحصائية. ولكن ، العلاقة بـين TFP في القطاع الزر اعي المصري وصادر ات المو اد الخام الزر اعية كانت سلبية وليست ذات دلالة إحصائية. أمسـا بالنسبة للتحليل (ءء سنة ) ، فإن العلاقة بين TFP في القطاع الزر اعي المصري وكل من صافي المعونــات الرسمية للتمية، استهالك الطاقة الكهربية، الواردات من السلع و الخدمات إيجابية وذات دلالة إحصائية. ولكن ، العلاقة بين TFP في القطاع الزر اعي المصري و استهلاك الوقود الحفري كانت سلبية وليست ذات دلالـــة إحصائية. أما بالنسبة للتحليل( م سنة) ، فإن العلاقة بين TFP في القطاع الزر اعي المــصري وكـل مــن خدمات النقل(ممثلة في خطوط السكك الحديدية)، و الاستثمار الاجنبي المباشر إيجابية وذات دلالـــة إحــصائية كبيرة. ولكن العلاقة بين TFP في القطاع الزر اعي المصري و الضرائب علي الصادر ات هي سلبية وليسـت ذات دلالة إحصائية. تبرز الار اسة : - أهمية سعر الصرف الرسمي و استقر اره النسبي في نمو TFP وكذلك الــواردات الـسلعية عنــدما تكــون التكنولوجيا منقدمة نسبياً حيث أن التجارة في هذه الحالة تعمل كحامل للمعرفة. - دعم الأنشطة الموجهة للتصدير التي تزيد من القيمة المضافة ، وليس المواد الخام. - إعادة توجيه وتشجيع المساعدات الخارجية الإنمائية الرسمية على الزر اعة ، ينبغي إيقاء القروض إلى الحد الأدنى من قيمها للحد من مستويات المديونية وعدم تحميلها على الديون الخارجية التقيلة. إن تحسين إدارة المساعدات الخارجية وتتسيقها سيزيدان من الأثر الإيجابي و الكفاءة لنظام المعونة. ســيؤدي رفــع الــدعم الفني و المالي إلى تحسين أداء المشروعات التي تمولها الجهات المانحة. كما أن الاستفادة القـصوى مــن الموارد المالية الخارجية ستؤثز إيجابيا على القدرة التتموية الوطنية لمصر . - المساعدات المجز أة التي تأتي في العديد من الثر ائح الصغيرة من عدد كبير من المانحين - تخلق تكــاليف معاملات مرتفعة وتجعل من الصعب على الدول الثريكة إدارة نتميتها بشكل فعال. كما أن تجزئة المعونة يزيد من خطر الازدو اجية و عدم كفاية توزيع المعونة بين المانحين. ايضـا تتفيذ خطط تطوير خاصة بكـلـ محافظة خاصة تلك التي نتلقى نسباً أقل من المساعدة. 
- البنية التحتية ليست مهمة فقط لنمو الإنتاجية بل إنها تثيرها أيضًا. ومع ذللك ، من المهــم مر اقبــة إدارتــهـ وتمويله عن كثب. إنتاج الكهرباء. لئه


محليا أو الممولة دوليا.

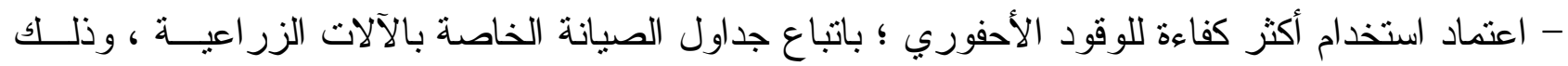



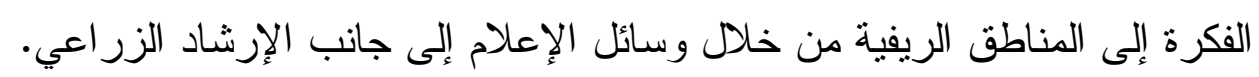

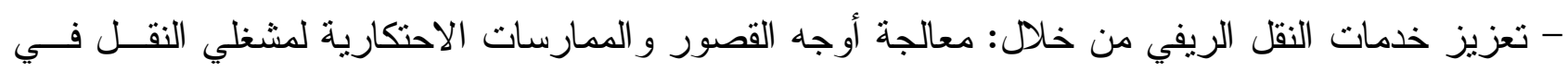

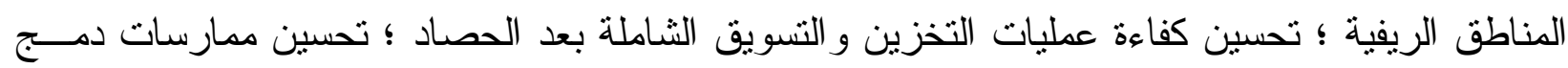

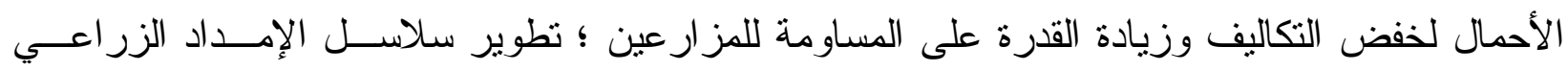

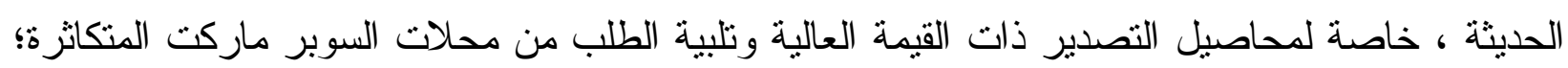

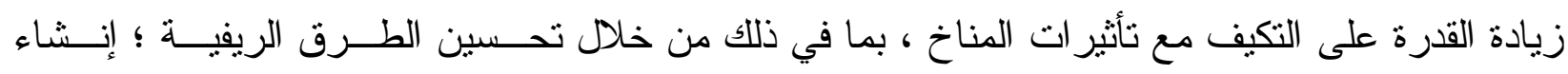

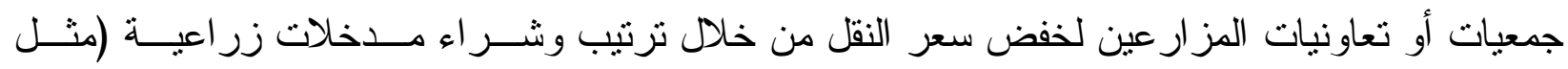
الأسمدة) بكميات كبيرة - تحسين الموارد المائية في المناطق الريفية من خلال. ا ـ ضمان إمدادات المياه لزر اعة آمنة

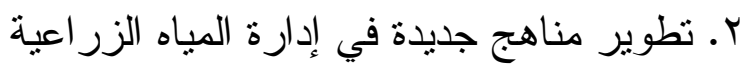
r. تطوير إدارة المباه الزر اعبة المناسبة للفقراء ع. تخفيف الآثار البيئية و الصحية للأنظمة الجديدة و القائمة

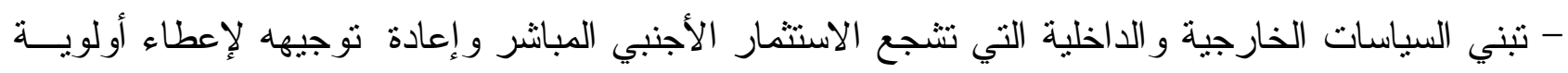
أكبر للتنديب على العمل الزر اعي وكذلك للمحافظات المهملة. - ولتجنب التداعيات السلبية لقيود التصدير الكمية مثل ضر ائب التصدير ، سيكون من المفيد وجــود إطــار

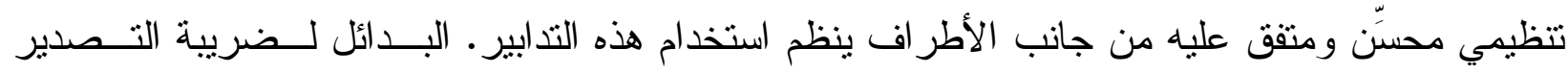


بصادرات إضافية بعد أن يفي المنتجون بمنطلبات المبيعات المحلية. 